International Journal of Modern Physics A

(C) World Scientific Publishing Company

\title{
HIGGSLESS DECONSTRUCTION WITHOUT BOUNDARY CONDITION *
}

\author{
HONG-JiAN He \\ Department of Physics, University of Texas at Austin, TX 78712, USA \\ (Email: hjhe@physics.utexas.edu)
}

Received: 26 November, 2004

\begin{abstract}
Deconstruction is a powerful means to explore the rich dynamics of gauge theories in four and higher dimensions. We demonstrate that gauge symmetry breaking in a compactified higher dimensional theory can be formulated via deconstructed 4D moose theory with spontaneous symmetry breaking and without boundary condition. The proper higher-D boundary conditions are automatically induced in the continuum limit rather than being imposed. We identify and analyze the moose theories which exhibit delayed unitarity violation (effective unitarity) as a collective effect of many gauge groups, without resorting to any known 5D geometry. Relevant phenomenological constraints are also addressed. hep-ph/0412113
\end{abstract}

Keywords: Deconstruction, Unitarity, Higgsless Electroweak Symmetry Breaking

\section{Advantageous Deconstruction}

Deconstruction 1 is a powerful means to explore the rich dynamics of gauge theories in four and higher dimensions. A compactified higher dimensional theory may be deconstructed into a proper moose representation ${ }^{2}$ or the equivalent transverse Wilson lattice ${ }^{3}$. An essential advantage of the deconstruction is that it allows us to formulate the often involved higher dimensional gauge symmetry breaking (without/with gauge group rank reduction) in terms of the conventional 4D gauged nonlinear sigma model à la CCWZ ${ }^{4}$, where no extra boundary condition $(\mathrm{BC})$ is required $a$ priori. Furthermore, deconstruction allows us to identify and analyze the general $4 \mathrm{D}$ moose theories with arbitrary inputs of gauge couplings $g_{j}$ and Goldstone decay constants $f_{k}$ or with only a few Kaluza-Klein $(\mathrm{KK}) 5$ modes. Such theories need not resemble any known higher-D geometry, but as we will show, they can still exhibit delayed unitarity violation (effective unitarity) as a collective effect of many participating gauge groups, which was originally revealed for the deconstruction of specified $5 \mathrm{D}$ geometries 67 . The effective unitarity in the compactified or deconstructed $5 \mathrm{D}$ Yang-Mills theories is ensured by the presence of spin-1 vector-bosons (gauge

* Presented at DPF-2004: Annual Meeting of the Division of Particles and Fields, American Physical Society, Riverside, California, USA, August 26-31, 2004. 
$K K$ modes 867 rather than the conventional Higgs scalar ${ }^{910}$, which is the key for seeking realistic Higgsless models of electroweak symmetry breaking (EWSB) in the recent literature 1112 . These new vector bosons also provide discovery signatures at the CERN LHC.

\section{Eaten Goldstone Bosons in General Higgsless Moose Theory}

Consider a most general linear moose theory, consisting of the replicated gauge group $G_{A}^{N+1} \otimes G_{B}^{M+1} \otimes G_{C}^{L+1} \otimes \cdots$ which is spontaneously broken to the diagonal subgroup $G_{D}$ by the link fields $U_{j}$ transforming as bi-fundamentals under the two adjacent gauge groups. Without losing generality, we will set 12$] G_{A}=S U(2)$, $G_{B}=U(1)$ and $G_{C}=\cdots=0$ for convenience of analyzing the EWSB, so the residual gauge symmetry is $G_{D}=U(1)_{\mathrm{em}}$ with a massless photon, as depicted in Fig. 1 12.

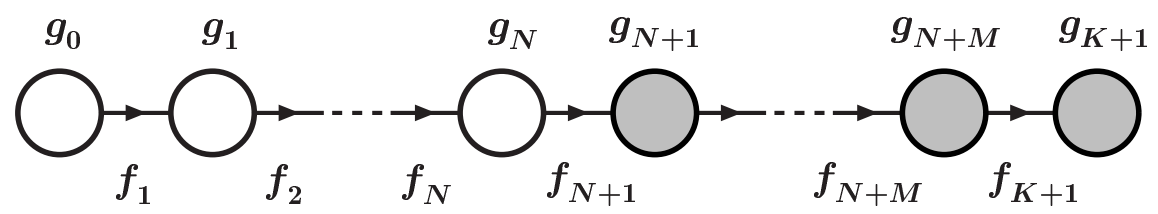

Fig. 1. A generic Higgsless deconstruction of linear moose theory with arbitrary product gauge group $G_{A}^{N+1} \otimes G_{B}^{M+1}$, where the most general inputs of gauge couplings and Goldstone decay constants $\left\{g_{j}, f_{k}\right\}(j=0,1, \cdots, K+1 ; k=1,2, \cdots, K+1 ; K \equiv N+M)$ are allowed. The open circles represent the group $G_{A}$ and shaded circles the group $G_{B}$.

The Lagrangian for this linear moose theory is

$$
\mathcal{L}=\sum_{j=0}^{K+1}-\frac{1}{2} \operatorname{Tr}\left(F_{j \mu \nu} F_{j}^{\mu \nu}\right)+\sum_{j=1}^{K+1} \frac{f_{j}^{2}}{4} \operatorname{Tr}\left[\left(D_{\mu} U_{j}\right)^{\dagger}\left(D^{\mu} U_{j}\right)\right],
$$

with $D^{\mu} U_{j}=\partial^{\mu} U_{j}-i g_{j-1} \mathbf{A}_{j-1}^{\mu} U_{j}+i g_{j} U_{j} \mathbf{A}_{j}^{\mu}$, and $U_{j}=\exp \left[i 2 \boldsymbol{\pi}_{j} / f_{j}\right]$, where $\mathbf{A}_{j}^{\mu} \equiv A_{j}^{a \mu} T^{a} \in S U(2)_{j}$ for $j=0,1, \cdots, N$, and $\mathbf{A}_{j}^{\mu} \equiv A_{j}^{\mu} T^{3} \in U(1)_{j}(j=N+$ $1, \cdots, K+1)$. Also, $\boldsymbol{\pi}_{j} \equiv \pi_{j}^{a} T^{a}(1 \leqslant j \leqslant N+1)$ and $\boldsymbol{\pi}_{j} \equiv \pi_{j}^{3} T^{3}(N+2 \leqslant j \leqslant K+1)$. The $(N+1) \times(N+1)$ mass matrix of charged gauge bosons is given by 12

$$
\mathbb{M}_{W}^{2}=\frac{1}{4}\left(\begin{array}{c|c|c|c|c}
g_{0}^{2} f_{1}^{2} & -g_{0} g_{1} f_{1}^{2} & & & \\
\hline-g_{0} g_{1} f_{1}^{2} & g_{1}^{2}\left(f_{1}^{2}+f_{2}^{2}\right) & -g_{1} g_{2} f_{2}^{2} & & \\
\hline & -g_{1} g_{2} f_{2}^{2} & g_{2}^{2}\left(f_{2}^{2}+f_{3}^{2}\right) & -g_{2} g_{3} f_{3}^{2} & \\
\hline & & \ddots & \ddots & \ddots \\
\hline & & & -g_{N-1} g_{N} f_{N}^{2} & g_{N}^{2}\left(f_{N}^{2}+f_{N+1}^{2}\right)
\end{array}\right),
$$


which may be denoted as $\mathbb{M}_{W}^{2}=\mathbb{M}_{[0, N+1)}^{2}$ by using the notation of open/closed intervals 12 . Similarly the $(K+2) \times(K+2)$ mass matrix of neutral gauge bosons is $\mathbb{M}_{Z}^{2}=\mathbb{M}_{[0, K+1]}^{2}$ and can be obtained from the matrix $\mathbb{M}_{[0, N+1)}^{2}$ with simple replacements, $N \rightarrow K+1$ and $f_{K+2} \rightarrow 0$. The eigenvalues of $\mathbb{M}_{W}^{2}$ are denoted by $M_{W}^{2}$ for light $W_{0}^{ \pm} \equiv W^{ \pm}$boson and $M_{W w}^{2}(w=1,2, \cdots, N)$ for heavy "KK" states $W_{w}^{ \pm}$, while $\mathbb{M}_{Z}^{2}$ has eigenvalues $0\left(M_{Z}^{2}\right)$ for photon $A_{\gamma}^{0}$ (light $Z_{0}^{0} \equiv Z^{0}$ boson) and $M_{Z z}^{2}(z=1,2, \cdots, K)$ for heavy "KK" states $Z_{z}^{0}$. With the diagonal matrices $\mathcal{F}_{n+1}=\operatorname{diag}\left(f_{1}, f_{2}, \cdots, f_{n+1}\right) / 2$ and $\mathcal{G}_{n+1}=\operatorname{diag}\left(g_{0}, g_{1}, \cdots, g_{n}\right)$, it is convenient to define the notations, $\mathbb{Q}_{W}=\mathcal{F}_{N+1} \mathcal{D}_{W} \mathcal{G}_{N+1}$ and $\mathbb{Q}_{Z}=\mathcal{F}_{K+1} \mathcal{D}_{Z} \mathcal{G}_{K+2}$, where the $(N+1) \times(N+1)$ and $(K+1) \times(K+2)$ non-diagonal matrices $\mathcal{D}_{W}$ and $\mathcal{D}_{Z}$ are
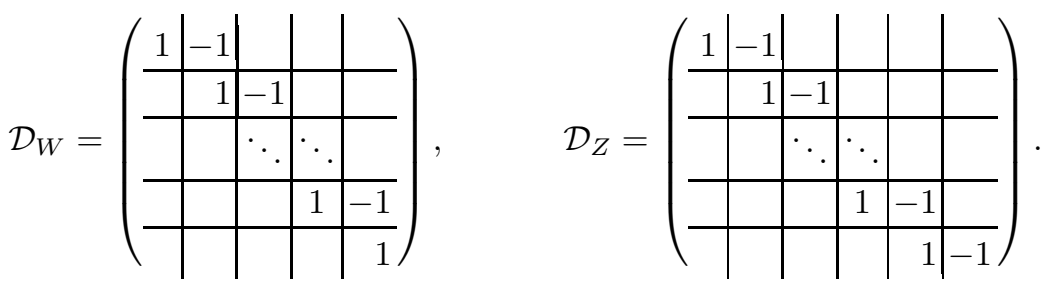

So the mass matrices $\left(\mathbb{M}_{W}^{2}, \mathbb{M}_{Z}^{2}\right)$ and their duals $\left(\widetilde{\mathbb{M}}_{W}^{2}, \widetilde{\mathbb{M}}_{Z}^{2}\right)$ can be written as 1213

$$
\mathbb{M}_{W}^{2}=\mathbb{Q}_{W}^{T} \mathbb{Q}_{W}, \quad \mathbb{M}_{Z}^{2}=\mathbb{Q}_{Z}^{T} \mathbb{Q}_{Z} ; \quad \widetilde{\mathbb{M}}_{W}^{2}=\mathbb{Q}_{W} \mathbb{Q}_{W}^{T}, \quad \widetilde{\mathbb{M}}_{Z}^{2}=\mathbb{Q}_{Z} \mathbb{Q}_{Z}^{T} ;
$$

where the dual mass matrices $\widetilde{\mathbb{M}}_{W}^{2}$ and $\widetilde{\mathbb{M}}_{Z}^{2}$ share the same nonzero eigenvalues as $\mathbb{M}_{W}^{2}$ and $\mathbb{M}_{Z}^{2}$, respectively. This is because $\mathbb{M}_{a}^{2}$ and $\widetilde{\mathbb{M}}_{a}^{2}(a=W, Z)$ are real symmetric matrices and $\mathbb{Q}_{a}$ can be diagonalized by the bi-orthogonal rotations,

$$
\widetilde{\mathbb{R}}_{a}^{T} \mathbb{Q}_{a} \mathbb{R}_{a}=\mathbb{Q}_{a}^{\text {diag }},
$$

which leads to

$$
\begin{aligned}
& \mathbb{R}_{a}^{T} \mathbb{M}_{a}^{2} \mathbb{R}_{a}=\left(\mathbb{Q}_{a}^{\text {diag }}\right)^{T}\left(\mathbb{Q}_{a}^{\text {diag }}\right) \equiv\left(\mathbb{M}_{a}^{2}\right)^{\text {diag }} \\
& \widetilde{\mathbb{R}}_{a}^{T} \widetilde{\mathbb{M}}_{a}^{2} \widetilde{\mathbb{R}}_{a}=\left(\mathbb{Q}_{a}^{\text {diag }}\right)\left(\mathbb{Q}_{a}^{\text {diag }}\right)^{T} \equiv\left(\widetilde{\mathbb{M}}_{a}^{2}\right)^{\text {diag }}
\end{aligned}
$$

The $(N+1) \times(N+1)$ matrices $\mathbb{M}_{W}^{2}$ and $\widetilde{\mathbb{M}}_{W}^{2}$ must have identical eigenvalues (which are all nonzero) because of $\left(\mathbb{M}_{W}^{2}\right)^{\text {diag }}=\left(\mathbb{Q}_{W}^{\text {diag }}\right)^{T}\left(\mathbb{Q}_{W}^{\text {diag }}\right)=\left(\mathbb{Q}_{W}^{\text {diag }}\right)\left(\mathbb{Q}_{W}^{\text {diag }}\right)^{T}=$ $\left(\widetilde{\mathbb{M}}_{W}^{2}\right)^{\text {diag }}$. On the other hand, the $(K+2) \times(K+2)$ matrix $\left(\mathbb{Q}_{Z}^{\text {diag }}\right)^{T}\left(\mathbb{Q}_{Z}^{\text {diag }}\right)=$ $\left(\mathbb{M}_{Z}^{2}\right)^{\text {diag }}$ has one zero-eigenvalue corresponding to the photon mass, and it is thus clear that the $(K+1) \times(K+1)$ matrix $\left(\mathbb{Q}_{Z}^{\text {diag }}\right)\left(\mathbb{Q}_{Z}^{\text {diag }}\right)^{T}=\left(\widetilde{\mathbb{M}}_{Z}^{2}\right)^{\text {diag }}$ has $K+1$ massive eigenvalues identical to those in $\left(\mathbb{M}_{Z}^{2}\right)^{\text {diag }}$.

Expanding the Lagrangian (1) gives the gauge-Goldstone boson mixing term, 12

$$
\begin{aligned}
\mathcal{L}_{\mathrm{GB}}^{\operatorname{mix}} & =\left[-\mathbb{A}_{\mu}^{+T} \mathbb{Q}_{W}^{T} \partial^{\mu} \Pi^{-}+\text {h.c. }\right]-\mathbb{A}_{\mu}^{3^{T}} \mathbb{Q}_{Z}^{T} \partial^{\mu} \Pi^{0} \\
& =\left[-\mathbb{W}_{\mu}^{+}{ }^{T} \mathbb{M}_{W}^{\operatorname{diag}} \partial^{\mu} \widetilde{\Pi}^{-}+\text {h.c. }\right]-\mathbb{Z}_{\mu}^{0} \widetilde{\mathbb{M}}_{Z}^{\operatorname{diag}} \partial^{\mu} \widetilde{\Pi}^{0},
\end{aligned}
$$


with

$$
\begin{array}{ll}
\mathbb{A}^{ \pm \mu}=\left(A_{0}^{ \pm \mu}, A_{1}^{ \pm \mu}, \cdots, A_{N}^{ \pm \mu}\right)^{T}, & \mathbb{A}^{3 \mu}=\left(A_{0}^{3 \mu}, A_{1}^{3 \mu}, \cdots, A_{K+1}^{3 \mu}\right)^{T} ; \\
\mathbb{W}^{ \pm \mu}=\left(W_{0}^{ \pm \mu}, W_{1}^{ \pm \mu}, \cdots, W_{N}^{ \pm \mu}\right)^{T}, & \mathbb{Z}^{0 \mu}=\left(Z_{0}^{0 \mu}, Z_{1}^{0 \mu}, \cdots, Z_{K}^{0 \mu}\right)^{T} ; \\
\Pi^{ \pm}=\left(\pi_{1}^{ \pm}, \pi_{2}^{ \pm}, \cdots, \pi_{N+1}^{ \pm}\right)^{T}, & \Pi^{0}=\left(\pi_{1}^{0}, \pi_{2}^{0}, \cdots, \pi_{K+1}^{0}\right)^{T} ; \\
\widetilde{\Pi}^{ \pm}=\left(\widetilde{\pi}_{0}^{ \pm}, \widetilde{\pi}_{1}^{ \pm}, \cdots, \widetilde{\pi}_{N}^{ \pm}\right)^{T}, & \widetilde{\Pi}^{0}=\left(\widetilde{\pi}_{0}^{0}, \widetilde{\pi}_{1}^{0}, \cdots, \widetilde{\pi}_{K}^{0}\right)^{T} ;
\end{array}
$$

where $\left(\mathbb{W}^{ \pm \mu}, \mathbb{Z}^{0 \mu}\right)$ are mass-eigenbasis fields, while $\left(\widetilde{\Pi}^{ \pm}, \widetilde{\Pi}^{0}\right)$ are "eaten" Goldstone fields, which are connected to the site Goldstone states $\left(\Pi^{ \pm}, \Pi^{0}\right)$ via

$$
\widetilde{\Pi}^{ \pm}=\widetilde{\mathbb{R}}_{W}^{T} \Pi^{ \pm}, \quad \widetilde{\Pi}^{0}=\widetilde{\mathbb{R}}_{Z}^{T} \Pi^{0} .
$$

Hence, the "eaten" Goldstones are exactly aligned with the "gauge boson" masseigenstates of the dual moose. The gauge-Goldstone mixing (17) can be removed by the familiar $R_{\xi}$ gauge-fixing term,

$$
\begin{aligned}
& \mathcal{L}_{\text {gf }}=\sum_{n=0}^{N}-\frac{1}{\xi_{W}} F_{n}^{+} F_{n}^{-}+\sum_{n=0}^{K+1}-\frac{1}{2 \xi_{Z}}\left(F_{n}^{0}\right)^{2}, \\
& F_{n}^{a}=\partial_{\mu} V_{n}^{a \mu}+\xi_{a} M_{a n} \widetilde{\pi}_{n}^{a},
\end{aligned}
$$

where $V_{n}^{a \mu} \in\left(W_{n}^{ \pm \mu}, Z_{n}^{0 \mu}\right), M_{a n} \in\left(M_{W n}, M_{Z n}\right)$ and $\xi_{a} \in\left(\xi_{W}, \xi_{Z}\right)$. Also, $Z_{K+1}^{0 \mu} \equiv$ $A_{\gamma}^{0 \mu}, M_{Z(K+1)} \equiv M_{\gamma}=0$ and $\tilde{\pi}_{K+1}^{0} \equiv 0$. From Eq. (10), the "eaten" Goldstone boson mass is given by $M_{\pi_{n}^{a}}^{2}=\xi_{a} M_{a n}^{2}$.

\section{4D Higgs Mechanism and Geometrization in 5D Continuum}

Corresponding to the gauge-fixing (10), we derive Faddeev-Popov ghost term,

$$
\mathcal{L}_{\mathrm{FP}}=\sum_{n=0}^{N}\left[\bar{c}_{n}^{+} \widehat{s} F_{n}^{-}+\bar{c}_{n}^{-} \widehat{s} F_{n}^{+}\right]+\sum_{n=0}^{K+1} \bar{c}_{n}^{Z} \widehat{s} F_{n}^{0},
$$

which ensures BRST 14 invariance at quantum level. Extending the notations of [15], we write the BRST transformations for the moose theory,

$$
\begin{array}{ll}
\widehat{s} c_{n}^{a}=-\frac{1}{2} g_{n} C^{a b c} c_{n}^{b} c_{n}^{c}, & \widehat{s} \bar{c}_{n}^{a}=-\xi_{a}^{-1} F_{n}^{a}, \\
\widehat{s} V_{n}^{a \mu}=D_{n b}^{a \mu}(V) c_{n}^{b}, & \widehat{s} \pi_{n}^{a}=D_{n b}^{a \pi}(\pi) c_{n}^{b}, \\
\widehat{s} \mathbb{V}^{a \mu}=\mathbb{R}_{a}^{T} \mathbb{D}_{b}^{a \mu} \mathbb{C}_{n}^{b}, & \widehat{s} \widetilde{\Pi}^{a}=\widetilde{\mathbb{R}}_{a}^{T} \mathbb{D}_{b}^{a \pi} \mathbb{C}_{n}^{b},
\end{array}
$$

where $\mathbb{V}^{a \mu}=\left(V_{0}^{a \mu}, \cdots, V_{P}^{a \mu}\right)^{T}, \widetilde{\Pi}^{a}=\left(\tilde{\pi}_{0}^{a}, \cdots, \tilde{\pi}_{P^{\prime}}^{a}\right)^{T}, \mathbb{C}^{a}=\left(c_{0}^{a}, \cdots, c_{P}^{a}\right)^{T}$, and $P, P^{\prime}=N\left(P=K+1, P^{\prime}=K\right)$ for charged (neutral) fields. In (12), $D_{n b}^{a \mu}(V)$ and $D_{n b}^{a \pi}(\pi)$ are given by the gauge transformations of $V_{n}^{a \mu}$ and $\pi_{n}^{a}$, respectively; also we define the matrix $\mathbb{D}_{b}^{i}=\operatorname{diag}\left(D_{0 b}^{i}, \cdots, D_{P b}^{i}\right)^{T}$. Following [6, 7], we directly 
extend Appendix-A of the fourth paper in 15] to derive a 4D Slavnov-Taylor (ST) identity for the moose theory (11) with replicated gauge group,

$$
\left\langle 0\left|T F^{a_{1} n_{1}}\left(x_{1}\right) F^{a_{2} n_{2}}\left(x_{2}\right) \cdots F^{a_{\ell} n_{\ell}}\left(x_{\ell}\right) \Phi_{\alpha}\right| 0\right\rangle=0,
$$

which is due to the manifest gauge invariance of the moose action $\int d x^{4} \mathcal{L}$ from (1) or the equivalent BRST invariance of the effective action $\int d x^{4}\left[\mathcal{L}+\mathcal{L}_{\text {gf }}+\mathcal{L}_{\mathrm{FP}}\right]$. In (13) $\Phi_{\alpha}$ denotes other possible amputated physical fields. We stress that (13) generally holds for any possible form of the gauge-fixing function $F^{a n}$. Compared to the SM case $\frac{15}{15}$, the main complication below comes from amputating the external fields $F_{n}^{a}$ in (13), due to the rotations in (5)-(6). We find it convenient to introduce a matrix notation for all relevant gauge-fixing functions,

$$
\mathbb{F}^{a}=\underline{\mathbb{K}}^{a T} \underline{\mathbb{V}}^{a}, \quad \underline{\mathbb{V}}^{a} \equiv\left(\begin{array}{c}
\mathbb{V}^{a \mu} \\
\widetilde{\Pi}^{a}
\end{array}\right), \quad \underline{\mathbb{K}}^{a} \equiv\left(\begin{array}{c}
\underline{I}_{a} \partial^{\mu} \\
\xi_{a} \underline{M}_{a}
\end{array}\right),
$$

where $\underline{I}_{a}=\operatorname{diag}(1, \cdots, 1)$ and $\underline{M}_{a}=\operatorname{diag}\left(M_{a 0}, \cdots, M_{a P}\right)$ are $(1+P) \times(1+P)$ diagonal matrices with $P=N(P=K+1)$ for charged (neutral) sector. In (14) we have extended the column $\widetilde{\Pi}^{a}$ to $1+P$ dimensional with $\widetilde{\pi}_{K+1}^{0} \equiv 0$. Introducing the external source term $\int d^{4} x\left[\underline{\mathbb{I}}^{a T} \underline{\mathbb{V}}^{a}+\overline{\mathbb{I}}^{a} \mathbb{C}^{a}+\overline{\mathbb{C}}^{a} \mathbb{I}^{a}\right]$, we derive a generating equation for connected Green functions,

$$
\underline{\mathbb{J}}^{a T}\left\langle 0\left|T \widehat{s} \underline{\mathbb{V}}^{a}(x)\right| 0\right\rangle+\overline{\mathbb{I}}^{a}(x)\left\langle 0\left|T \widehat{s} \mathbb{C}^{a}(x)\right| 0\right\rangle-\left\langle 0\left|T \widehat{s} \overline{\mathbb{C}}^{a}(x)\right| 0\right\rangle \mathbb{I}^{a}(x)=0,
$$

from which we deduce an ST identity for the matrix propagator of $\underline{\mathbb{V}}^{a}$,

$$
\underline{\mathbb{K}}^{a T} \underline{\mathcal{D}}^{a b}(p)=-\left[\underline{\Omega}^{a b}\right](p)^{T},
$$

with

$$
\begin{aligned}
& \underline{\mathcal{D}}^{a b}(p)=\left\langle 0\left|T \underline{\mathbb{V}}^{a} \underline{\mathbb{V}}^{b^{T}}\right| 0\right\rangle(p), \quad \mathcal{S}(p) \delta^{a b}=\left\langle 0\left|T \mathbb{C}^{a} \overline{\mathbb{C}}^{b}\right| 0\right\rangle(p), \\
& \underline{\Omega}^{a b}(p) \equiv \underline{\widehat{\Omega}}^{a b}(p) \mathcal{S}(p), \\
& \underline{\widehat{\Omega}}^{a b}(p) \equiv\left\langle 0\left|T \widehat{s} \underline{\mathbb{V}}^{b}\right| \overline{\mathbb{C}}^{a}\right\rangle(p) \equiv\left(\begin{array}{c}
\left\langle 0\left|T \widehat{s} \mathbb{V}^{b \mu}\right| \overline{\mathbb{C}}^{a}\right\rangle \\
\left\langle 0\left|T \widehat{s} \widetilde{\Pi}^{b}\right| \overline{\mathbb{C}}^{a}\right\rangle
\end{array}\right)_{(p)} \equiv\left(\begin{array}{c}
-i p^{\mu} \delta^{a b} \mathbb{R}_{a}^{T}\left[\mathbf{1}+\Delta_{V}^{a}\left(p^{2}\right)\right] \\
\delta^{a b} \widetilde{\mathbb{R}}_{a}^{T} \mathbb{Q}_{a}\left[\mathbf{1}+\Delta_{\pi}^{a}\left(p^{2}\right)\right]
\end{array}\right) .
\end{aligned}
$$

Using (16) and collecting $F^{a n}$ in the matrix form $\mathbb{F}^{a}$ for each external line in (13), we make an amputation for $[\mathbf{1 3}), 0=G\left[\mathbb{F}^{a}(p), \cdots\right]=-\left[\underline{\Omega}^{a b}\right]^{T} \mathcal{T}\left[\underline{\mathbb{V}}^{a}(p), \cdots\right]$, which leads to

$$
\begin{aligned}
0 & =\mathcal{T}\left[p_{\mu} \mathbb{V}^{a \mu}(p)-\widetilde{\mathbb{M}}_{a}^{\operatorname{diag}} \mathbf{C}^{a} \widetilde{\Pi}^{a}(p), \cdots \cdots\right], \\
\mathbf{C}^{a} & \equiv-i\left(\mathbb{Q}_{a}^{T} \widetilde{\mathbb{R}}_{a}\right)^{-1}\left(\mathbf{1}+\Delta_{V}^{a}\left(p^{2}\right)^{T}\right)^{-1}\left(\mathbf{1}+\Delta_{\pi}^{a}\left(p^{2}\right)^{T}\right)\left(\mathbb{Q}_{a}^{T} \widetilde{\mathbb{R}}_{a}\right) \\
& =-i[\mathbf{1}+O(\text { loop })] .
\end{aligned}
$$

Repeating this amputation for all external lines in (13), we arrive at a matrix identity for $S$-matrix elements,

$$
\mathcal{T}\left[\overline{\mathbb{F}}^{a_{1}}\left(p_{1}\right), \overline{\mathbb{F}}^{a_{2}}\left(p_{2}\right), \cdots, \overline{\mathbb{F}}^{a_{\ell}}\left(p_{\ell}\right), \Phi_{\alpha}\right]=0,
$$


where $\overline{\mathbb{F}}^{a}(p) \equiv p^{\mu} \mathbb{V}_{\mu}^{a}-\widetilde{\mathbb{M}}_{a}^{\operatorname{diag}} \mathbf{C}^{a} \widetilde{\Pi}^{a}=\widetilde{\mathbb{M}}_{a}^{\operatorname{diag}}\left(\mathbb{V}_{S}^{a}-\mathbf{C}^{a} \widetilde{\Pi}^{a}\right)$ with $\mathbb{V}_{S}^{a}=\epsilon_{S}^{\mu} \mathbb{V}_{\mu}^{a}$ $\left(\epsilon_{S}^{\mu} \equiv p^{\mu} / \widetilde{\mathbb{M}}_{a}^{\text {diag }}\right), \mathbb{V}^{a \mu}=\mathbb{W}^{ \pm \mu}, \mathbb{Z}^{0 \mu}$, and $\mathbf{C}^{a}=-i[1+O$ (loop) $]$. The identity (19) states that in the $S$-matrix element the unphysical eaten "KK" Goldstones $\widetilde{\Pi}^{a}$ and the unphysical "KK" scalar gauge-components $\mathbb{V}_{S}^{a}$ are confined, so they together have no net contribution to any physical process - a quantitative formulation of the 4D Higgs mechanism at the $S$-matrix level, which holds even without a physical Higgs boson such as in the present moose theory (gauged nonlinear sigma model) with replicated gauge groups. Under high energy expansion, Eq. (19) results in a generalized form of the equivalence theorem (ET) 156 , which connects the high energy longitudinal gauge boson scattering amplitude to that of the "eaten" Goldstone bosons,

$$
\mathcal{T}\left[V_{L}^{a_{1} n_{1}}, V_{L}^{a_{2} n_{2}}, \cdots\right]=C_{\bmod }^{n_{1} m_{1}, n_{2} m_{2} \cdots} \mathcal{T}\left[\widetilde{\pi}^{a_{1} m_{1}}, \widetilde{\pi}^{a_{2} m_{2}}, \cdots\right]+O\left(\frac{M_{a n}}{E_{n}}\right),
$$

where $V_{n}^{a}=W_{n}^{ \pm}, Z_{n}^{0}(n=0,1,2, \cdots)$, and sums over repeated indices $\left(m_{1}, m_{2}, \cdots\right)$ are implied. The exact expression of the $O\left(M_{a n} / E_{n}\right)$ term is obtained from directly expanding the ET identity (19), in the same way as in [15]. The radiative modification factor is

$$
C_{\bmod }^{n_{1} m_{1}, n_{2} m_{2} \cdots} \equiv \mathbf{C}_{n_{1} m_{1}}^{a_{1}} \mathbf{C}_{n_{2} m_{2}}^{a_{2}} \cdots=(-i)^{\ell}\left[\left(\delta_{n_{1} m_{1}} \delta_{n_{2} m_{2}} \cdots\right)+O(\text { loop })\right]
$$

which extends Ref. 15] to the case of replicated gauge group.

Analyzing both sides of (20), we observed $\underline{6}$ that the non-canceled leading $E^{2}$ terms in the usual nonlinear gauged sigma model are now suppressed by large $N$ due to the collective effect of many "KK" modes in the deconstruction theory.

To take the 5D continuum limit we redefine the lattice link field (Wilson line),

$$
U_{j}(x)=U\left(x, x_{j}^{5}\right)=\exp \left[i \int_{x_{j-1}^{5}}^{x_{j}^{5}} d x^{5} g_{j} \mathbf{A}_{5}\left(x, x^{5}\right)\right]=\exp \left[i \mathbf{a} g_{j} \mathbf{A}_{5}\left(x, x_{j}^{5}\right)\right]
$$

where $\mathbf{a}=\Delta x_{j}^{5}$ is lattice spacing and $A_{5}^{a}\left(x, x_{j}^{5}\right)$ is related to the site-Goldstone boson $\pi_{j}^{a}(x)$ in (11), $A_{5}^{a}\left(x, x_{j}^{5}\right)=\pi_{j}^{a}(x)\left(2 / g_{j} f_{j} \mathbf{a}\right)$. So the site-Goldstone field $\pi_{j}^{a}$ will geometrize as $\widehat{A}_{5}^{a}$ component of the $5 \mathrm{D}$ gauge field $\widehat{A}_{J}^{a}=\left(\widehat{A}_{\mu}^{a}, \widehat{A}_{5}^{a}\right)$, where $J, I, \ldots \in(\mu, 5)$ denote the $5 \mathrm{D}$ Lorentz indices. Following [16] and ignoring the $U(1)^{M+1}$ part of Fig. 1 for simplicity, we define the position-dependent couplings $g_{j}=g_{5} \kappa_{j} / \sqrt{\mathbf{a}}$ and decay constants $f_{j}=h_{j} f$, where $g_{5}$ and $f$ are pure constants of mass-dimension $-\frac{1}{2}$ and 1 , respectively. Thus we can derive, $D^{\mu} U_{j}=-i \mathbf{a} g_{j} F_{j}^{a \mu 5}+O\left(\mathbf{a}^{2}\right)=-i \mathbf{a} g_{5} \widehat{F}^{a \mu 5}\left(x, x_{j}^{5}\right)+O\left(\mathbf{a}^{2}\right)$, where the identification $A_{j}^{a \mu, 5}(x) \rightarrow\left(\sqrt{\mathbf{a}} / \kappa_{j}\right) \widehat{A}^{a J}\left(x, x_{j}^{5}\right)=\left(g_{5} / g_{j}\right) \widehat{A}^{a J}\left(x, x_{j}^{5}\right)$ is made under $\mathbf{a}=L / N \rightarrow 0$. As such, we see that the Goldstone Lagrangian in the second term of (11) just gives the continuum $\widehat{A}_{5}^{a}$-Lagrangian, $\int_{0}^{L} d x^{5}\left[-\frac{1}{2} h^{2}\left(x^{5}\right) \widehat{F}^{a \mu 5} \widehat{F}_{\mu 5}^{a}\right]$, after imposing a normalization condition $\mathbf{a}=\left(2 / g_{5} f\right)^{2}$. With these, we reproduce a bulk $S U(2)_{5 \mathrm{D}}$ gauge theory from (I) in the continuum limit,

$$
S_{5}=\int d^{5} \widehat{x} \sqrt{-\widehat{g}} \widehat{g}^{I K} \widehat{g}^{J L} \frac{-1}{4 \kappa^{2}\left(x^{5}\right)} \widehat{F}_{I J}^{a} \widehat{F}_{K L}^{a},
$$


defined on a general 5D background,

$$
d s^{2}=\left[\kappa\left(x^{5}\right) h\left(x^{5}\right)\right]^{2} \eta_{\mu \nu} d x^{\mu} d x^{\nu}-d x^{5} d x^{5},
$$

where the metric $\widehat{g}_{I K}=\operatorname{diag}\left(\eta_{\mu \nu}(\kappa h)^{2},-1\right)$. For the simplest case $\kappa=h=1$, it reduces to flat 5D geometry, while for the case $\kappa=1$ and $h=\exp \left[-k\left|x^{5}\right|\right]$ it reduces to the familiar warped $\mathrm{RS} 1 \mathrm{17}$. Various extended continuum models from (23) can be derived from the moose theory (11) by including the $U(1)^{M+1}$ groups and/or proper folding(s) of the moose chain in Fig. 1. These together with the induced 5D BCs and possible brane kinetic terms will be analyzed in Sec. 4.

Analyzing (23) we construct an $R_{\xi}$ gauge-fixing term to remove the $\widehat{A}^{a \mu}-\widehat{A}^{a 5}$ mixing,

$$
\widehat{\mathcal{L}}_{\mathrm{gf}}=-\frac{1}{2 \xi}\left(\widehat{F}^{a}\right)^{2}, \quad \widehat{F}^{a}=\kappa^{-1} \partial_{\mu} \widehat{A}^{a \mu}+\xi \kappa \partial_{5}\left(h^{2} \widehat{A}^{a 5}\right),
$$

The corresponding ghost term is given by

$$
\widehat{\mathcal{L}}_{\mathrm{FP}}=\widehat{\bar{c}} \widehat{s} \widehat{F}^{a},
$$

and the 5D BRST transformations are

$$
\widehat{s} \widehat{A}^{a J}=D_{b}^{a J}(\widehat{A}) \widehat{c}^{b}, \quad \widehat{s c}^{a}=-\frac{1}{2} g_{5} C^{a b c} \widehat{c} \widehat{c}^{c}, \quad \widehat{s} \widehat{\bar{c}}^{a}=-\xi^{-1} \widehat{F}^{a} .
$$

Using 5D gauge invariance of the action $S_{5}$, we derive an ST identity 7 in parallel to the $4 \mathrm{D}$ result (13),

$$
\left\langle 0\left|T \widehat{F}^{a_{1}}\left(\widehat{x}_{1}\right) \widehat{F}^{a_{2}}\left(\widehat{x}_{2}\right) \cdots \widehat{F}^{a_{\ell}}\left(\widehat{x}_{\ell}\right) \widehat{\Phi}_{\alpha}\right| 0\right\rangle=0 .
$$

The $5 \mathrm{D}$ equation of motion (EOM) for free field $\widehat{A}_{\mu 5}^{a}$ is

$$
\begin{aligned}
& \left(\partial_{\mu}^{2}-\kappa^{2} \partial_{5} h^{2} \partial_{5}\right) \widehat{A}_{\nu}^{a}-\left(1-\xi^{-1}\right) \partial_{\nu} \partial_{\mu} \widehat{A}^{a \mu}=0, \\
& \partial_{5}\left(\kappa^{2} \partial_{5} h^{2} \widehat{A}_{5}^{a}\right)-\xi^{-1} \partial_{\mu}^{2} \widehat{A}_{5}^{a}=0,
\end{aligned}
$$

where we have made integration by part in the action $S_{5}+S_{\mathrm{gf}}+S_{\mathrm{FP}}$ and verified that all surface terms vanish under the induced consistent BCs in Sec.4. Then, we define the KK expansions,

$$
\widehat{A}^{a \mu}(\widehat{x})=\frac{1}{\sqrt{L}} \sum_{n=0}^{\infty} V_{n}^{a \mu}(x) \mathcal{X}_{n}\left(x^{5}\right), \quad \widehat{A}^{a 5}(\widehat{x})=\frac{1}{\sqrt{L}} \sum_{n=0}^{\infty} V_{n}^{a 5}(x) \widetilde{\mathcal{X}}_{n}\left(x^{5}\right) .
$$

As in [18] we find it convenient to work in the momentum space for $4 \mathrm{D}$ KK fields $V_{n}^{a \mu, 5}$ and position space for $5 \mathrm{D}$ wavefunction $\left(\mathcal{X}_{n}, \widetilde{\mathcal{X}}_{n}\right)$. Imposing the $4 \mathrm{D}$ EOMs for the free KK fields as usual, $\left(\partial_{\mu}^{2}+M_{a n}^{2}\right) V_{n}^{a \nu}(x)-\left(1-\xi^{-1}\right) \partial^{\mu} \partial^{\nu} V_{n \mu}^{a}(x)=0$ and $\left(\partial_{\mu}^{2}+\widetilde{M}_{a n}^{2}\right) V_{n}^{a 5}(x)=0$, we derive from (29),

$$
-\kappa^{2} \partial_{5}\left(h^{2} \partial_{5} \mathcal{X}_{n}\right)=M_{a n}^{2} \mathcal{X}_{n}, \quad-\partial_{5}\left(\kappa^{2} \partial_{5} h^{2} \widetilde{\mathcal{X}}_{n}\right)=\xi^{-1} \widetilde{M}_{a n}^{2} \widetilde{\mathcal{X}}_{n}
$$


where $\left(M_{a n}, \widetilde{M}_{a n}\right)$ are mass-eigenvalues of $4 \mathrm{D}$ KK fields $\left(V_{n}^{a \mu}, V_{n}^{a 5}\right)$. Acting $\partial_{5}$ on the first equation of (31) we see, $\widetilde{\mathcal{X}}_{n} \propto \partial_{5} \mathcal{X}_{n}$ and $\widetilde{M}_{a n}^{2}=\xi M_{a n}^{2}$. So, with the normalization conditions of $\left(\mathcal{X}_{n}, \widetilde{\mathcal{X}}_{n}\right)$, we deduce from (31),

$$
\partial_{5} \mathcal{X}_{n}=M_{a n} \widetilde{\mathcal{X}}_{n}, \quad \partial_{5}\left(h^{2} \widetilde{\mathcal{X}}_{n}\right)=-\kappa^{-2} M_{a n} \mathcal{X}_{n} .
$$

With these we decompose the 5D gauge-fixing function $\widehat{F}^{a}$ as

$$
\begin{aligned}
\widehat{F}^{a}(\widehat{x}) & =\frac{1}{\sqrt{L}} \sum_{n=0}^{\infty} F^{a n}(x) \mathcal{X}_{n}\left(x^{5}\right) / \kappa\left(x^{5}\right), \\
F^{a n}(x) & =\partial_{\mu} V_{n}^{a \mu}(x)-\xi M_{a n} V_{n}^{a 5}(x) \equiv \underline{K}_{a n}^{T} \underline{V_{n}^{a}},
\end{aligned}
$$

where $\underline{K}_{a n}=\left(\partial_{\mu},-\xi M_{a n}\right)^{T}$ and $\underline{V}_{n}^{a}=\left(V_{n}^{a \mu}, V_{n}^{a 5}\right)^{T}$. Thus we derive a 4D ST identity from (28),

$$
\left\langle 0\left|T F^{a_{1} n_{1}}\left(x_{1}\right) F^{a_{2} n_{2}}\left(x_{2}\right) \cdots F^{a_{\ell} n_{\ell}}\left(x_{\ell}\right) \Phi_{\alpha}\right| 0\right\rangle=0 .
$$

Adding the KK expansions for ghost fields,

$$
\widehat{c}^{a}(\widehat{x})=\frac{1}{\sqrt{L}} \sum_{n=0}^{\infty} c_{n}^{a}(x) \mathcal{X}_{n}\left(x^{5}\right), \quad \widehat{\bar{c}}^{a}(\widehat{x})=\frac{1}{\sqrt{L}} \sum_{n=0}^{\infty} \bar{c}_{n}^{a}(x) \mathcal{X}_{n}\left(x^{5}\right) / \kappa\left(x^{5}\right),
$$

we derive the BRST transformations for the KK fields,

$$
\begin{array}{ll}
\widehat{s} V_{n}^{a \mu}=D_{n m}^{a b, \mu} c_{m}^{b}, & \widehat{s} V_{n}^{a 5}=D_{n m}^{a b, 5} c_{m}^{b}, \\
\widehat{s} c_{n}^{a}=-\frac{1}{2} g C^{a b c} \mathfrak{D}_{n}^{m \ell} c_{m}^{b} c_{\ell}^{c}, & \widehat{s} \bar{c}_{n}^{a}=-\xi^{-1} F^{a n},
\end{array}
$$

where

$$
\begin{aligned}
& D_{n m}^{a b, \mu}=-\delta^{a b} \delta_{n m} \partial^{\mu}+\left[\frac{1}{L} \int_{0}^{L} d x^{5} \kappa^{-2} \mathcal{X}_{n} \mathcal{X}_{m} \mathcal{X}_{\ell}\right] g C^{a b c} V_{\ell}^{c \mu}, \\
& D_{n m}^{a b, 5}=-\delta^{a b} \delta_{n m} M_{a m}+\left[\frac{1}{L} \int_{0}^{L} d x^{5} h^{2} \widetilde{\mathcal{X}}_{n} \mathcal{X}_{m} \widetilde{\mathcal{X}}_{\ell}\right] g C^{a b c} V_{\ell}^{c 5}, \\
& \mathfrak{D}_{n}^{m \ell}=\frac{1}{L} \int_{0}^{L} d x^{5} \kappa^{-2} \mathcal{X}_{n} \mathcal{X}_{m} \mathcal{X}_{\ell},
\end{aligned}
$$

and repeated indices are summed up. To amputate the external fields $F^{a n}$ in (34), we deduce an ST identity for the KK propagator of $V_{n}^{a}$, similar to Eq. (16),

$$
\underline{K}_{a n}^{T} \underline{\mathcal{D}}_{n m}^{a b}(p)=-\left[\underline{\Omega}_{n m}^{a b}\right](p)^{T},
$$

with

$$
\begin{aligned}
& \mathcal{D}_{n m}^{a b}(p)=\left\langle 0\left|T \underline{V_{n}^{a}} \frac{V_{m}^{b}}{b^{T}}\right| 0\right\rangle(p), \quad \mathcal{S}_{n m}(p) \delta^{a b}=\left\langle 0\left|T c_{n}^{a} \bar{c}_{m}^{b}\right| 0\right\rangle(p), \\
& \underline{\Omega}_{n m}^{a b}(p) \equiv \underline{\widehat{\Omega}}_{m j}^{a b}(p) \mathcal{S}_{j n}(p), \\
& \underline{\widehat{\Omega}}_{m j}^{a b}(p) \equiv\left\langle 0\left|T \widehat{\widehat{s}} \underline{V_{m}^{b}}\right| \bar{c}_{j}^{a}\right\rangle(p) \equiv\left(\begin{array}{c}
\left\langle 0\left|T \widehat{s} V_{m}^{b \mu}\right| \bar{c}_{j}^{a}\right\rangle \\
\left\langle 0\left|T \widehat{s} V_{m}^{b 5}\right| \bar{c}_{j}^{a}\right\rangle
\end{array}\right)_{(p)} \equiv\left(\begin{array}{c}
-i p^{\mu} \delta^{a b}\left[\delta_{j m}+\Delta_{j m}^{a}\left(p^{2}\right)\right] \\
-M_{a m} \delta^{a b}\left[\delta_{j m}+\widetilde{\Delta}_{j m}^{a}\left(p^{2}\right)\right]
\end{array}\right) .
\end{aligned}
$$


Extension of the above formulation to include possible brane term is straightforward. Consider, for simplicity, a brane term similar to (60)- (61) in Sec. 4 , at $y=0$, or $L$,

$S_{\mathrm{BT}}=\int d^{5} \widehat{x} \delta\left(x^{5}-y\right) \frac{-\zeta}{4} \widehat{F}^{a \mu \nu} \widehat{F}_{\mu \nu}^{a}=\int d^{5} \widehat{x} \delta\left(x^{5}-y\right) \sqrt{-\widehat{g}} \widehat{g}^{I K} \widehat{g}^{J L} \frac{-\widehat{\zeta}}{4 \kappa^{2}} \widehat{F}_{I J}^{a} \widehat{F}_{K L}^{a}$,

where $\widehat{\zeta}=\zeta \kappa^{2}(y)$. In (40) the second expression is equivalent to the first one under the covariant BC $\widehat{F}_{\mu 5}^{a}=0$ [cf. (153)], and appears more convenient for direct extension of the above derivation. The brane term (40) may vary, depending on the residual symmetry at the boundary. We stress that the total action $S_{5}+S_{\mathrm{BT}}$ is invariant under the $5 \mathrm{D}$ gauge symmetry supplemented by the $\mathrm{BCs}$ [which are induced from the continuum limit of the gauge-invariant moose theory (1), cf. Sec. 4]. The quantized action may be written as $S_{5}+S_{\mathrm{BT}}+S_{\mathrm{gf}}+S_{\mathrm{FP}}$ with $S_{\mathrm{gf}}+S_{\mathrm{FP}}=\int d^{5} \widehat{x}\left[1+\widehat{\zeta} \delta\left(x^{5}-\right.\right.$ $y)]\left(\widehat{\mathcal{L}}_{\mathrm{gf}}+\widehat{\mathcal{L}}_{\mathrm{FP}}\right)$, which is expected from the deconstruction viewpoint since the gauge group at each lattice site of the $5 \mathrm{D}$ (including boundary sites) can be quantized with its own gauge-fixing and ghost terms as shown earlier in this section. With these and a prescription of the vanishing total derivative $\partial_{5}(\cdots)$ under the integration $\int d x^{5}\left[1+\widehat{\zeta} \delta\left(x^{5}-y\right)\right]$, we readily verify that the above Eqs. (27)-(39) remain, except that all integrals in (37) will contain an extra brane-term-factor $\left[1+\widehat{\zeta} \delta\left(x^{5}-y\right)\right]$. Accordingly, the $5 \mathrm{D}$ wavefunctions $\left(\mathcal{X}_{n}, \widetilde{\mathcal{X}}_{n}\right)$ satisfy the normalization conditions,

$$
\begin{aligned}
L^{-1} \int d x^{5}\left[1+\widehat{\zeta} \delta\left(x^{5}-y\right)\right] \kappa^{-2} \mathcal{X}_{n} \mathcal{X}_{m} & =\delta_{n m}, \\
L^{-1} \int d x^{5}\left[1+\widehat{\zeta} \delta\left(x^{5}-y\right)\right] h^{2} \widetilde{\mathcal{X}}_{n} \widetilde{\mathcal{X}}_{m} & =\delta_{n m},
\end{aligned}
$$

on the general 5D background. The corresponding normalization conditions for the KK mass-terms are,

$$
\begin{aligned}
L^{-1} \int d x^{5}\left[1+\widehat{\zeta} \delta\left(x^{5}-y\right)\right] h^{2} \partial_{5} \mathcal{X}_{n} \partial_{5} \mathcal{X}_{m} & =M_{a n}^{2} \delta_{n m}, \\
L^{-1} \int d x^{5}\left[1+\widehat{\zeta} \delta\left(x^{5}-y\right)\right] \kappa^{2} \partial_{5}\left(h^{2} \widetilde{\mathcal{X}}_{n}\right) \partial_{5}\left(h^{2} \widetilde{\mathcal{X}}_{m}\right) & =M_{a n}^{2} \delta_{n m} .
\end{aligned}
$$

Under Eq. (32) we see that (42) consistently reduces to (41).

With these given, we are ready to amputate the external fields in (34) and derive an identity for $S$-matrix elements,

$$
\mathcal{T}\left[\bar{F}^{a_{1} n_{1}}\left(p_{1}\right), \bar{F}^{a_{2} n_{2}}\left(p_{2}\right), \cdots, \bar{F}^{a_{\ell} n_{\ell}}\left(p_{\ell}\right), \Phi_{\alpha}\right]=0,
$$

where $\bar{F}^{a n}=p^{\mu} V_{\mu}^{a n}-M_{a n} \widehat{C}_{n m}^{a} V_{5}^{a m}=M_{a n}\left(V_{S}^{a n}-\widehat{C}_{n m}^{a} V_{5}^{a m}\right), V_{S}^{a n}=\epsilon_{S}^{\mu} V_{\mu}^{a n}$ $\left[\epsilon_{S}^{\mu} \equiv k^{\mu} / M_{a n}=\epsilon_{L}^{\mu}-v^{\mu}, v^{\mu}=O\left(M_{a n} / E_{n}\right)\right]$, and $\widehat{C}_{n m}^{a} \equiv i\left(M_{a m} / M_{a n}\right)[(\mathbf{1}+$ $\left.\left.\widetilde{\Delta}^{a}\left(p^{2}\right)\right)\left(\mathbf{1}+\Delta^{a}\left(p^{2}\right)\right)^{-1}\right]_{m n}$. Similar to (19) for the deconstruction theory, our ET identity (43) shows that the unphysical scalar-KK-component $V_{S}^{a n}$ and the fifth gauge-KK-component $V_{5}^{a n}$ (or its linear composition $\widehat{C}_{n m}^{a} V_{5}^{a m}$ ) are confined at the $S$-matrix level, so they together have zero contribution to any physical process. This is just the quantitative $S$-matrix formulation of the 5D geometric Higgs mechanism $(\mathrm{GHM}) \stackrel{\sqrt{78}}{ }$, where $V_{5}^{\text {an }}$ 's serve as the would-be Goldstone bosons and get converted 
to the longitudinal gauge KK-modes $V_{L}^{a n}$ 's. Expanding the ET identity [43], we thus derive a general Kaluza-Klein ET (KK-ET ${ }^{\mathbb{8}}$ ) at asymptotic energy,

$$
\mathcal{T}\left[V_{L}^{a_{1} n_{1}}, V_{L}^{a_{2} n_{2}}, \cdots\right]=\widehat{C}_{\bmod }^{n_{1} m_{1}, n_{2} m_{2} \cdots} \mathcal{T}\left[V_{5}^{a_{1} m_{1}}, V_{5}^{a_{2} m_{2}}, \cdots\right]+O\left(\frac{M_{a n}}{E_{n}}\right)
$$

where $\widehat{C}_{\bmod }^{n_{1} m_{1}, n_{2} m_{2} \cdots}=\widehat{C}_{n_{1} m_{1}}^{a_{1}} \cdots \widehat{C}_{n_{\ell} m_{\ell}}^{a_{\ell}}=i^{\ell}\left[\left(\delta_{n_{1} m_{1}} \delta_{n_{2} m_{2}} \cdots\right)+O(\right.$ loop $\left.)\right]$, and the external momenta are put on-shell $\left(p_{n}^{2}=M_{a n}^{2}\right)$. The exact expression of the suppressed term $O\left(M_{a n} / E_{n}\right)$ in (44) is tedious and is directly obtained from expanding the ET identity (43) by $\epsilon_{S}^{\mu}=\epsilon_{L}^{\mu}-v^{\mu}$, in the same way as in [15]..$^{1}$ We stress that our KK-ET (44) and its KK-ET identity (43) are valid for arbitrary geometry with any consistent $B C$ and possible brane term such as (40) (cf. Sec. 4 for detail). ${ }^{2}$ The KK-ET (44) is the manifestation of the 5D geometric Higgs mechanism $\frac{78}{4}$ at the $S$-matrix level, where the conversion $V_{5}^{a n} \Longrightarrow V_{L}^{a n}$ is realized. An essential advantage of this $5 \mathrm{D}$ formulation is that we start with the well-defined $4 \mathrm{D}$ moose theory (gauged nonlinear sigma model) which is manifestly gauge-invariant without any BC. The consistent BCs and possible brane terms are automatically induced by taking proper continuum limits (cf. Sec.4), which also ensure the gauge-invariance of the $5 \mathrm{D}$ action at the boundaries.

The gauge interactions of $V_{5}^{a n}$ arise from $\int d^{5} \widehat{x} \frac{-h^{2}}{2} \widehat{F}_{\mu 5}^{a} \widehat{F}^{a \mu 5}$ under KK expansion. Examining this 87 and applying power counting 19 , we find that scalar amplitude $\mathcal{T}\left[V_{5}^{a_{1} n_{1}}, V_{5}^{a_{2} n_{2}}, \ldots \cdots\right]=O\left(E^{0}\right)$ generally holds 7 , independent of the detail of any particular compactification/BC and possible brane term. Because of this and Eq. (44), we conclude that the longitudinal amplitude $\mathcal{T}\left[V_{L}^{a_{1} n_{1}}, V_{L}^{a_{2} n_{2}}, \cdots \cdots\right]=$ $O\left(E^{0}\right)$, generally guaranteeing the $E$-power cancellations down to a constant.

Finally, we note that the nonlinearly realized gauge symmetry in the moose theories may be regarded as a (spontaneously) broken phase formulation, but the crucial difference from an arbitrary or random explicit symmetry breaking is that our formalism (including the continuum 5D action) is manifestly gauge-invariant or BRST-invariant in which the would-be Goldstone bosons and the corresponding "Higgs" mechanism are consistently embedded, so the various Slavnov-Taylor and Ward-Takahashi identities can be derived even in the broken phase 20 . It is such general identities and their resulting ET (20) or KK-ET (44) that have directly guaranteed the relevant $E$-cancellations in all $2 \rightarrow n(n \geqslant 2)$ longitudinal gauge boson scatterings for any $4 \mathrm{D}$ or compactified higher-D theory, leading to the effective unitarity $\underline{687}$

\footnotetext{
${ }^{1}$ As well-knowr 15877, the exact ET identity 43) ensures the sum of the Goldstone amplitude and the lengthy $O\left(M_{a n} / E_{n}\right)$ term to precisely equal the amplitude of longitudinal gauge bosons.

${ }^{2}$ After the completion of this manuscript, a preprint ${ }^{28}$ appeared which considered a generalization of the 5D ET in Refs. 87 to include brane term in the special case of flat $S^{1} / Z_{2}$.
} 


\section{Deconstruction, (Non)Geometric Gauge Symmetry Breaking and Induced Boundary Conditions}

Various compactified 5D theories can be derived by taking proper continuum limit of our general moose theory (Fig. 1). The bulk gauge symmetry and its breaking at the boundary are essentially determined by the structure of $4 \mathrm{D}$ moose and its spontaneous symmetry breaking pattern. In comparison with the traditional 4D Higgs mechanism 9 which spontaneously breaks the gauge symmetry by nontrivial vacuum, the $5 \mathrm{D}$ gauge symmetry can be spontaneously broken by the proper compactification at boundaries which arise from the "boundary" groups (sites) of the moose theory (Fig. 1) under possible folding(s). This provides us a conceptually clean and elegant formulation of the gauge symmetry breaking (without/with gauge-group rank reduction) in compactified 5D theories, without inputting the BCs a priori or relying on the technique of adding extra boundary Higgs fields 21 . Our generic moose analysis below also reveals that the induced 5D BCs for $\widehat{A}^{a \mu}$ do not depend on particular choice of bulk geometry, while the same is not generally true for $\widehat{A}^{a 5}$.

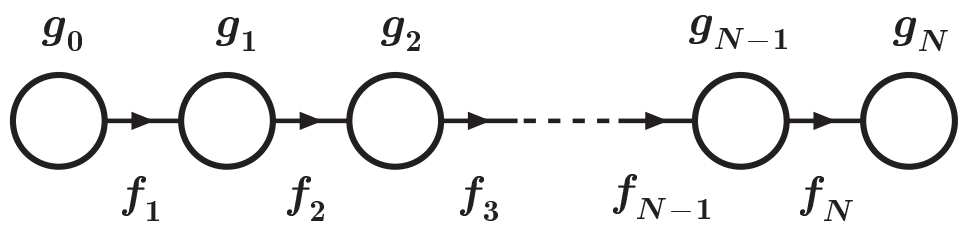

Fig. 2. Higgsless Moose-A: a special case of Fig. 1 with $M=0$ and $f_{N+1}=0$. The gauge symmetry breaking pattern is $S U(2)^{N+1} \rightarrow S U(2)_{D}$, with massless zero modes.

We start by considering the special case of Fig. 1 with $M=0$ and $f_{N+1}=0$, whose gauge symmetry breaking pattern is $S U(2)^{N+1} \rightarrow S U(2)_{D}$, as shown in Fig. 2. The $(N+1) \times(N+1)$ gauge boson mass-matrix $\mathbb{M}_{a}^{2}=\mathbb{M}_{[0, N]}^{2}$ is given in (2) with $f_{N+1}=0$, and its orthonormal eigenvectors are denoted as $\mathbb{X}_{n}=$ $\left(X_{0 n}, X_{1 n}, \cdots, X_{N n}\right)^{T}(n=0,1,2, \cdots, N)$. So the gauge-eigenbasis field $\mathbb{A}^{a \mu}$ and mass-eigenbasis field $\mathbb{V}^{a \mu}$ are connected by $\mathbb{A}^{a \mu}=\mathbb{R}_{a} \mathbb{V}^{a \mu}$ with rotation $\mathbb{R}_{a}=$ $\left(\mathbb{X}_{0}, \mathbb{X}_{1}, \cdots, \mathbb{X}_{N}\right)$, i.e.,

$$
A_{j}^{a \mu}=\sum_{n=0}^{N} V_{n}^{a \mu} X_{j n}, \quad \Longrightarrow \quad \widehat{A}^{a \mu}(\widehat{x})=\frac{1}{\sqrt{L}} \sum_{n=0}^{\infty} V_{n}^{a \mu}(x) \mathcal{X}_{n}\left(x^{5}\right),
$$

where the second relation is the corresponding continuum KK-expansion under $N \rightarrow \infty$ and $j \mathbf{a} \rightarrow x^{5}$. Analyzing the eigenvalue equation $\mathbb{M}_{a}^{2} \mathbb{X}_{n}=\lambda_{n} \mathbb{X}_{n}$, we find that the eigenvectors satisfy the following consistency relations,

$$
g_{0} X_{0, n}-g_{-1} X_{-1, n}=0, \quad g_{N+1} X_{N+1, n}-g_{N} X_{N, n}=0 .
$$

As shown above Eq. (23), taking the 5D continuum limit leads to the identification, $A_{j}^{a \mu}(x) \rightarrow\left(g_{5} / g_{j}\right) \widehat{A}^{a \mu}\left(x, x_{j}^{5}\right)$. Combining this with (45) we find that the eigenvector 
$X_{j n}$ is related to the $5 \mathrm{D}$ KK-wavefunction $\mathcal{X}_{n}\left(x^{5}\right)$ via $g_{j} X_{j n} \rightarrow\left(g_{5} / \sqrt{L}\right) \mathcal{X}_{n}\left(x_{j}^{5}\right)$. Hence, we rewrite (46) as conditions for the $5 \mathrm{D}$ KK-wavefunction $\mathcal{X}_{n}\left(x_{j}^{5}\right)$,

$$
\mathcal{X}_{n}\left(x_{0}^{5}\right)-\mathcal{X}_{n}\left(x_{-1}^{5}\right)=0, \quad \mathcal{X}_{n}\left(x_{N+1}^{5}\right)-\mathcal{X}_{n}\left(x_{N}^{5}\right)=0 .
$$

From (47), we thus derive the induced boundary conditions (BCs) of Neumann type in the $5 \mathrm{D}$ continuum limit,

$$
\left.\partial_{5} \widehat{A}_{\mu}^{a}\right|_{x^{5}=0}=0,\left.\quad \partial_{5} \widehat{A}_{\mu}^{a}\right|_{x^{5}=L}=0,
$$

where $L=N \mathbf{a}$ is the length of 5D. This compactified 5D theory has an unbroken $S U(2)$ gauge symmetry survived in $4 \mathrm{D}$ (corresponding to the residual diagonal group $S U(2)_{D}$ of the Moose-A in Fig. 2), which ensures the massless zero KK-modes. So, the Neumann BCs (48) break a 5D $S U(2)$ into a 4D $S U(2)$ (with massive gauge KK towers as well as massless zero modes), and preserve the rank of 5D gauge group, precisely matching the symmetry breaking structure of its parent Moose-A in Fig. 2.

We can further derive the BCs for $\widehat{A}^{a 5}$. In the continuum limit the site-Goldstone fields $\pi_{j}^{a}$ will geometrize as $\widehat{A}^{a 5}$ [cf. (22)], while the eaten Goldstones $\widetilde{\pi}_{n}^{a}$ will geometrize as the KK modes $V_{n}^{a 5}$ [cf. (50) below]. From the mass-diagonalization in Eqs. (6) and (9) and the $R_{\xi}$ gauge-fixing in Eq. (10), we see that the eaten Goldstone fields $\widetilde{\Pi}^{a}$ have mass matrix $\xi_{a}\left(\widetilde{\mathbb{M}}_{a}^{2}\right)^{\text {diag }}$ and the site-Goldstones $\Pi^{a}$ have mass matrix $\xi_{a} \widetilde{\mathbb{M}}_{a}^{2}$. For the Moose-A (Fig. 2), we derive the $N \times N$ mass matrix $\widetilde{\mathbb{M}}_{a}^{2}$,

$$
\widetilde{\mathbb{M}}_{a}^{2}=\frac{1}{4}\left(\begin{array}{c|c|c|c|c}
\left(g_{0}^{2}+g_{1}^{2}\right) f_{1}^{2} & -g_{1}^{2} f_{1} f_{2} & & & \\
\hline-g_{1}^{2} f_{1} f_{2} & \left(g_{1}^{2}+g_{2}^{2}\right) f_{2}^{2} & -g_{2}^{2} f_{2} f_{3} & & \\
\hline & -g_{2}^{2} f_{2} f_{3} & \left(g_{2}^{2}+g_{3}^{2}\right) f_{3}^{2} & -g_{3}^{2} f_{3} f_{4} & \\
\hline & & \ddots & \ddots & \ddots \\
\hline & & & -g_{N-1}^{2} f_{N-1} f_{N} & \left(g_{N-1}^{2}+g_{N}^{2}\right) f_{N}^{2}
\end{array}\right),
$$

whose orthonormal eigenvectors are denoted by $\widetilde{\mathbb{X}}_{n}=\left(\widetilde{X}_{1 n}, \widetilde{X}_{2 n}, \cdots, \widetilde{X}_{N n}\right)^{T}$ $(n=1,2, \cdots, N)$. The orthogonal rotation matrix in (9) is thus given by $\mathbb{R}_{a}=$ $\left(\widetilde{\mathbb{X}}_{1}, \widetilde{\mathbb{X}}_{2}, \cdots, \widetilde{\mathbb{X}}_{N}\right)$. So, in the continuum limit we have the following geometrization,

$$
\pi_{j}^{a}=\sum_{n=0}^{N} \widetilde{\pi}_{n}^{a} \widetilde{X}_{j n}, \quad \Longrightarrow \quad \widehat{A}^{a 5}(\widehat{x})=\frac{1}{\sqrt{L}} \sum_{n=0}^{\infty} V_{n}^{a 5}(x) \widetilde{\mathcal{X}}_{n}\left(x^{5}\right) .
$$

As shown below Eq. (22), the site-Goldstone field $\pi_{j}^{a}=\left(g_{j} f_{j} \mathbf{a} / 2\right) A_{5}^{a}\left(x, x_{j}^{5}\right)$ is connected to the $5 \mathrm{D}$ gauge field $\widehat{A}_{5}^{a}\left(x, x_{j}^{5}\right)$ via $\pi_{j}^{a} \rightarrow\left(\mathbf{a} g_{5} f_{j} / 2\right) \widehat{A}_{5}^{a}\left(x, x_{j}^{5}\right)$ in the continuum limit. So, from (50) we find that $\widetilde{X}_{j n}$ and $\widetilde{\mathcal{X}}_{n}\left(x_{j}^{5}\right)$ are connected via $\widetilde{X}_{j n} \rightarrow\left(\mathbf{a} g_{5} f_{j} / 2 \sqrt{L}\right) \widetilde{\mathcal{X}}_{n}\left(x_{j}^{5}\right)$. Analyzing the eigenvalue equation $\widetilde{\mathbb{M}}_{a}^{2} \widetilde{X}_{n}=\lambda_{n} \widetilde{X}_{n}$, we find that the eigenvectors must satisfy the following consistency conditions,

$$
\widetilde{X}_{0, n}=0, \quad \tilde{X}_{N+1, n}=0 ; \quad \Longrightarrow \quad \widetilde{\mathcal{X}}_{n}\left(x_{0}^{5}\right)=0, \quad \widetilde{\mathcal{X}}_{n}\left(x_{N+1}^{5}\right)=0 .
$$


In the continuum limit, they result in the induced 5D BCs for $\widehat{A}_{5}^{a}$,

$$
\left.\widehat{A}_{5}^{a}\right|_{x^{5}=0}=0,\left.\quad \widehat{A}_{5}^{a}\right|_{x^{5}=L}=0,
$$

which are both of Dirichlet type, unlike the Neumann BCs for $\widehat{A}^{a \mu}$ in (48). This is fully consistent with the relation $\partial_{5} \mathcal{X}_{n} \propto \widetilde{\mathcal{X}}_{n}$ in (32) for 5D KK-wavefunctions. We also note that the Neumann BCs (48) for $\widehat{A}^{a \mu}$ and the Dirichlet BCs (52) for $\widehat{A}^{a 5}$ can be combined into a gauge-covariant form,

$$
\left.\widehat{F}_{\mu 5}^{a}\right|_{x^{5}=0}=0,\left.\quad \widehat{F}_{\mu 5}^{a}\right|_{x^{5}=L}=0,
$$

which explicitly retains the gauge-invariance of the $5 \mathrm{D}$ action at the corresponding boundary.

We then turn to a different gauge symmetry breaking structure. This is most cleanly described by the Moose-B in Fig. 3 (which we called "enlarged moose" in $2001^{17}$ ). This is a special case of our general moose theory in Fig. 1 with $M=0$ and $g_{N+1}=0$, with gauge group breaking pattern $S U(2)^{N+1} \rightarrow$ nothing. As we will show, it induces the Dirichlet type BCs in 5D continuum limit and reduces the rank of the 5D gauge group.

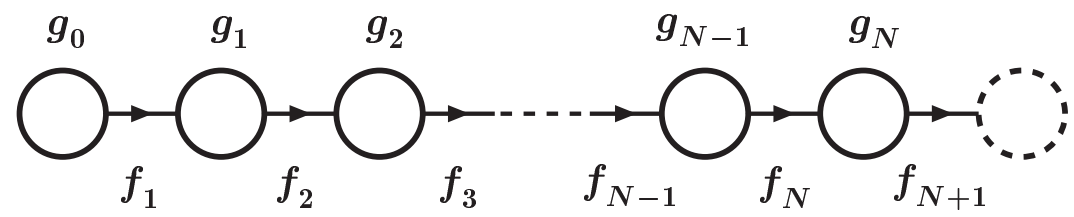

Fig. 3. Higgsless Moose-B: a special case of Fig. 1 with $M=0$ and $g_{N+1}=0$. The gauge symmetry breaking pattern is $S U(2)^{N+1} \rightarrow$ nothing, without massless "zero modes".

The gauge boson mass matrix $\mathbb{M}_{a}^{2}=\mathbb{M}_{[0, N+1)}^{2}$ is just given by Eq. (2]), which has massive eigenvalues only. Analyzing the eigenvalue equation $\mathbb{M}_{a} \mathbb{X}_{n}=\lambda_{n} \mathbb{X}_{n}$, we observe that the eigenvectors satisfy the following consistency relations,

$$
\begin{aligned}
g_{0} X_{0, n}-g_{-1} X_{-1, n}=0, & X_{N+1, n}=0 ; \\
\Longrightarrow \quad \mathcal{X}_{n}\left(x_{0}^{5}\right)-\mathcal{X}_{n}\left(x_{-1}^{5}\right)=0, & \mathcal{X}_{n}\left(x_{N+1}^{5}\right)=0 .
\end{aligned}
$$

Taking the continuum limit as before, we derive the induced BCs,

$$
\left.\partial_{5} \widehat{A}_{\mu}^{a}\right|_{x^{5}=0}=0,\left.\quad \widehat{A}_{\mu}^{a}\right|_{x^{5}=L}=0
$$

where we see that similar to (48) the $\mathrm{BC}$ at $x^{5}=0$ remains the Neumann type, but the new condition at $x^{5}=L$ is a Dirichlet $\mathrm{BC}$. The moose representation of Fig. 3 clearly shows that it is this induced Dirichlet BC at $x^{5}=L$ that completely breaks the $5 \mathrm{D} S U(2)$ gauge group and makes all zero KK-modes massive. The above formulation based on the Moose-A and -B can be directly generalized to any $5 \mathrm{D}$ gauge theory with an arbitrary simple group $G_{A}$. 
We have also derived the BCs for $\widehat{A}^{a 5}$ in the Moose-B. Consider the mass matrix $\xi_{a} \widetilde{\mathbb{M}}_{a}^{2}$ for site-Goldstone fields $\Pi^{a}$, where the $(N+1) \times(N+1)$ matrix $\widetilde{\mathbb{M}}_{a}^{2}$ can be obtained from (49) by simple replacements $N \rightarrow N+1$ and $g_{N+1} \rightarrow 0$. Analyzing the eigenvalue equation $\widetilde{\mathbb{M}}_{a}^{2} \widetilde{\mathbb{X}}_{n}=\lambda_{n} \widetilde{\mathbb{X}}_{n}$, we deduce the following consistency relations for the eigenvectors,

$$
\tilde{X}_{0, n}=0, \quad f_{N+2} \widetilde{X}_{N+2, n}-f_{N+1} \widetilde{X}_{N+1, n}=0 .
$$

As before, we have the identification, $\widetilde{X}_{j n} \rightarrow\left(\mathbf{a} g_{5} f_{j} / 2 \sqrt{L}\right) \widetilde{\mathcal{X}}_{n}\left(x_{j}^{5}\right)$, in the continuum limit. So we can rewrite (56) as conditions for $\widetilde{\mathcal{X}}_{n}\left(x_{j}^{5}\right)$,

$$
\widetilde{\mathcal{X}}_{n}\left(x_{0}^{5}\right)=0, \quad f_{N+2}^{2} \widetilde{\mathcal{X}}_{n}\left(x_{N+2}^{5}\right)-f_{N+1}^{2} \widetilde{\mathcal{X}}_{n}\left(x_{N+1}^{5}\right)=0 .
$$

With the definition $f_{j}=h_{j} f=h\left(x_{j}^{5}\right) f$, we derive the induced $B C s$ from (56) in the $5 \mathrm{D}$ continuum limit,

$$
\left.\widehat{A}_{5}^{a}\right|_{x^{5}=0}=0,\left.\quad \partial_{5}\left(h^{2} \widehat{A}_{5}^{a}\right)\right|_{x^{5}=L}=0 .
$$

They are fully consistent with the BCs for $\widehat{A}_{\mu}^{a}$ in (55) according to Eq. (32) which gives $\partial_{5} \mathcal{X}_{n} \propto \widetilde{\mathcal{X}}_{n}$ and $\partial_{5}\left(h^{2} \widetilde{\mathcal{X}}_{n}\right) \propto \mathcal{X}_{n}$. Note that, unlike the Dirichlet BC for $\widehat{A}_{5}^{a}$, the Neumann BC for $\widehat{A}_{5}^{a}$ does depend on the bulk geometry via the function $h\left(x^{5}\right)$. Implications of this will be explored elsewhere 26 .

(a).

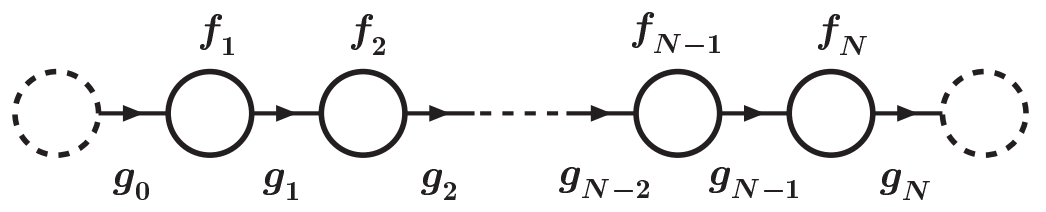

(b).

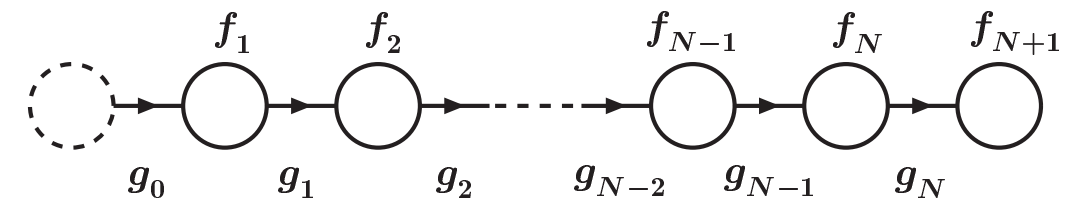

Fig. 4. Two dual mooses: (a). Moose- $\widetilde{A}$ is the dual of Moose-A in Fig. 2; (b). Moose- $\widetilde{B}$ is the dual of Moose-B in Fig. 3.

It is also instructive to view the Goldstone mass matrix $\widetilde{\mathbb{M}}_{\Omega}^{2}$ as the "gauge boson" mass matrix generated in the corresponding dual moose 1312 the exchange $\left\{g_{n}\right\} \longleftrightarrow\left\{f_{n}\right\}$ from the original moose. We show the dual versions of the Moose-A and -B in Fig. 4. With the notations of open/closed intervals, it may be convenient to denote the dual mass matrix as $\widetilde{\mathbb{M}}_{a}^{2}=\widetilde{\mathbb{M}}_{(0, N+1)}^{2}$ for Moose- $\widetilde{\mathrm{A}}$ (where the sites $j=0, N+1$ themselves have no contribution due to $f_{0}=f_{N+1}=0$ ), and $\widetilde{\mathbb{M}}_{a}^{2}=\widetilde{\mathbb{M}}_{(0, N+1]}^{2}$ for Moose- $\widetilde{\mathrm{B}}$ (where the site $j=0$ has no contribution due 
to $f_{0}=0$ ). Comparing the dual Moose- $\widetilde{\mathrm{A}}$ [Fig. 4(a)] with Moose-A [Fig. 2], we can now intuitively see why the BCs for $\widetilde{\mathbb{X}}_{n}$ and $\widehat{A}^{a 5}$ in (51)-(52) just take Dirichlet type, different from the Neumann BCs (46)-(48) for $\mathbb{X}_{n}$ and $\widehat{A}^{a \mu}$. Similarly, comparison of the dual Moose- $\widetilde{B}$ [Fig. 4(b)] with Moose-B [Fig. 3] explains the difference between the BCs (57)-(58) and (54)-(55).

Another advantage of our general deconstruction formalism is that we can also derive the proper brane kinetic terms in the continuum limit. Considering our Moose-B for instance, we quantify how a brane term at $x^{5}=0$ is reconstructed from localizing the boundary group $S U(2)_{0}$ at site $j=0$. Setting $f_{1}^{2} \gg f_{j}^{2}(j \geqslant 2)$ for taking the continuum limit (which prevents the site-0 from joining the $5 \mathrm{D}$ geometry), we examine the equation of motion (EOM) for $A_{0}^{a \mu}$ and derive the following relation,

$$
A_{0}^{a \mu}=\left(g_{1} / g_{0}\right) A_{1}^{a \mu} .
$$

In the continuum theory, this localizes $S U(2)_{0}$ as a brane kinetic term at $x^{5}=0$,

$$
S_{\mathrm{BT}}=\int d^{5} \widehat{x} \delta\left(x^{5}-0\right) \frac{-\left(\widehat{g} / g_{0}\right)^{2}}{4} \widehat{F}_{\mu \nu}^{a} \widehat{F}^{a \mu \nu},
$$

where $\widehat{g} \equiv g_{5}$ denotes the $5 \mathrm{D}$ gauge coupling. The above brane term may be viewed as being actually localized at $x^{5}=0^{+}$, the right-neighborhood of the point $x^{5}=0$ at which our BC $\left.\partial_{5} \widehat{A}_{\mu}^{a}\right|_{x^{5}=0}=0$ is imposed. This is because our deconstruction procedure of deriving this BC from (54) includes the boundary site $j=0$ itself plus its left-neighborhood. We also note that the theory obeys a covariant $\mathrm{BC} \widehat{F}_{5 \mu}^{a}=0$ at $x^{5}=0$ [similar to (53)], so we may rewrite the brane term (60) to have explicit invariance under the $5 \mathrm{D}$ gauge group at $x^{5}=0$,

$$
S_{\mathrm{BT}}=\int d^{5} \widehat{x} \delta\left(x^{5}-0\right) \sqrt{-\widehat{g}} \widehat{g}^{I K} \widehat{g}^{J L} \frac{-\widehat{\zeta}}{4 \kappa^{2}} \widehat{F}_{I J}^{a} \widehat{F}_{K L}^{a},
$$

where $\widehat{\zeta}=\left(\widehat{g} / g_{0}\right)^{2} \kappa^{2}(0)$. Other brane terms may be rewritten in the similar way.

Next, we analyze the Moose-C in Fig. 5, which is a special case of Fig. 1 with $M=0$ and $g_{N+1} \neq 0$. As a slight variation of Moose-B, Moose-C has gauged its subgroup $U(1)_{N+1}$ and has the symmetry breaking pattern $S U(2)^{N+1} \otimes U(1) \rightarrow$ $U(1)_{\mathrm{em}}$, as a straightforward extension of Ref. [22] which studied the nontrivial minimal models $S U(2)^{3} \otimes U(1) \rightarrow U(1)_{\mathrm{em}}(N+1=3)$ and $S U(2)^{2} \otimes U(1)^{2} \rightarrow$ $U(1)_{\mathrm{em}}(N+1=M+1=2)$ with general inputs $\left(g_{j}, f_{k}\right)$.

The charged gauge boson mass matrix $\mathbb{M}_{W}^{2}=\mathbb{M}_{[0, N+1)}^{2}$ is given in (2), the same as in Moose-B, while the neutral gauge boson mass matrix $\mathbb{M}_{Z}^{2}=\mathbb{M}_{[0, N+1]}^{2}$ can be obtained from $\mathbb{M}_{Z}^{2}[$ Moose-A] with a replacement $N \rightarrow N+1$. In consequence we find that the consistency relations for the eigenvectors $\left\{\mathbb{X}_{n}^{ \pm}\right\}$and $\left\{\mathbb{X}_{n}^{3}\right\}$ are just a mixture of Moose-A and -B in (46) and (54),

$$
\begin{array}{ll}
g_{0} X_{0, n}^{ \pm}-g_{-1} X_{-1, n}^{ \pm}=0, & X_{N+1, n}^{ \pm}=0 ; \\
g_{0} X_{0, n}^{3}-g_{-1} X_{-1, n}^{3}=0, & g_{N+2} X_{N+2, n}^{3}-g_{N+1} X_{N+1, n}^{3}=0 .
\end{array}
$$




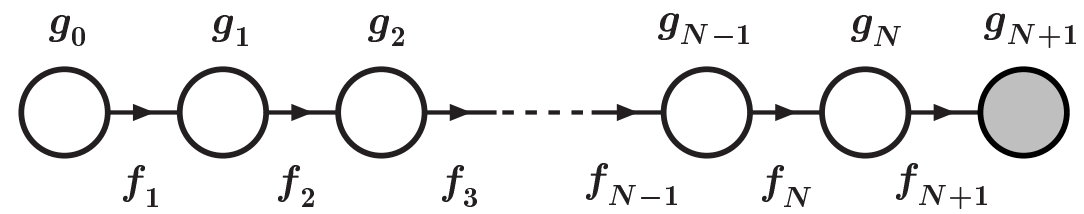

Fig. 5. Higgsless Moose-C: a special case of Fig. 1 with $M=0$ and $g_{N+1} \neq 0$. The gauge symmetry breaking pattern is $S U(2)^{N+1} \otimes U(1) \rightarrow U(1)_{\mathrm{em}}$, extended from Ref. [22].

So, with the same reasoning as before, we obtain the induced continuum BCs which combine (55) for charged sector and (48) for neutral sector,

$$
\left.\partial_{5} \widehat{A}_{\mu}^{ \pm}\right|_{x^{5}=0}=0,\left.\quad \partial_{5} \widehat{A}_{\mu}^{3}\right|_{x^{5}=0}=0 ;\left.\quad \widehat{A}_{\mu}^{ \pm}\right|_{x^{5}=L}=0,\left.\quad \partial_{5} \widehat{A}_{\mu}^{3}\right|_{x^{5}=L}=0 .
$$

In the continuum limit, the boundary group $U(1)_{N+1}$ will be localized at $x^{5}=$ $L$, similar to the brane term which we reconstructed in (60). So, we can localize $U(1)_{N+1}$ by setting $f_{N+1}^{2} \gg f_{j}^{2}(j \leqslant N)$ for the continuum limit. We quantify this by examining the EOM for $A_{N+1}^{3 \mu}$ under large $f_{N+1}^{2}$ limit, thus we derive a relation,

$$
A_{N+1}^{3 \mu}=\left(g_{N} / g_{N+1}\right) A_{N}^{3 \mu},
$$

which generates the localized brane kinetic term at $x^{5}=L$,

$$
S_{\mathrm{BT}}=\int d^{5} \widehat{x} \delta\left(x^{5}-L\right) \frac{-\left(\widehat{g} / g_{N+1}\right)^{2}}{4} \widehat{F}_{\mu \nu}^{3} \widehat{F}^{3 \mu \nu} .
$$

This brane term is actually localized at $x^{5}=L^{-}$, the left-neighborhood of $x^{5}=L$ at which the BC $\left.\partial_{5} \widehat{A}_{\mu}^{3}\right|_{x^{5}=L}=0$ is imposed, since our deconstruction procedure of deriving this BC from (62) includes the boundary site $j=N+1$ plus its rightneighborhood.

We then consider the Moose-D in Fig. 6 which is obtained from Fig. 1 by folding the $S U(2)$ lattice chain once at the middle with renumbering $N \rightarrow 2 N$ and setting $M=0$. The gauge symmetry breaking structure is $S U(2)_{L}^{N+1} \otimes S U(2)_{R}^{N} \otimes U(1) \rightarrow$ $U(1)_{\mathrm{em}}$, where $S U(2)_{L}^{N+1}$ part has inputs $\left\{g_{j}, f_{k}\right\}$ and $S U(2)_{R}^{N} \otimes U(1)$ part has inputs $\left\{\widetilde{g}_{j}, \widetilde{f}_{k}\right\}$.

The continuum limit of Moose-D has the bulk gauge group $\left[S U(2)_{L} \otimes S U(2)_{R}\right]_{5 \mathrm{D}}$ (with $5 \mathrm{D}$ gauge couplings $\widehat{g}_{L}$ and $\widehat{g}_{R}$ ), plus a $U(1)_{0}$ brane term localized at $x^{5}=$ 0 [similar to (65) of Moose-C]. The other brane terms at $x^{5}=0$ for $S U(2)_{L}$ [similar to (60) of Moose-C] and at $x^{5}=L$ for $S U(2)_{D}$ are also possible. From our moose formulation, it is clear that at $x^{5}=0$ the BCs for $S U(2)_{L}$ and $S U(2)_{R}$ are essentially the same as those of Moose- $\mathrm{C}$ at $x^{5}=0$ and $x^{5}=L$, respectively, i.e.,

$$
\left.\partial_{5} \widehat{A}_{L}^{a \mu}\right|_{x^{5}=0}=0 ;\left.\quad \widehat{A}_{R}^{ \pm \mu}\right|_{x^{5}=0}=0,\left.\quad \partial_{5} \widehat{A}_{R}^{3 \mu}\right|_{x^{5}=0}=0 .
$$

We then derive the new BCs at $x^{5}=L$. Ordering the sites $j=0,1, \cdots, N$, $\widetilde{N}, \widetilde{N+1}, \cdots, \widetilde{1}, \widetilde{0}$, we can write down the charged gauge boson mass matrix $\mathbb{M}_{W}^{2}=$ 


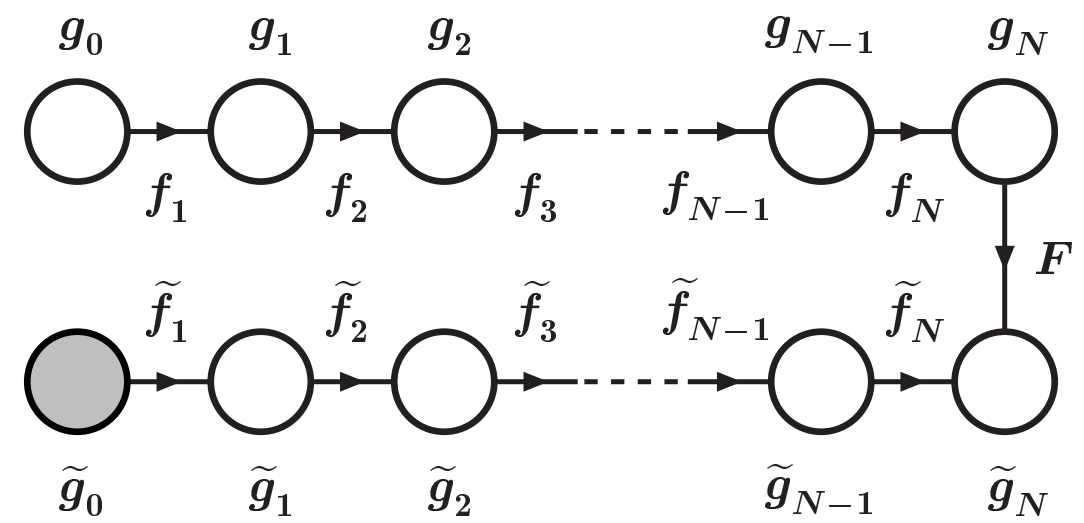

Fig. 6. Higgsless Moose-D: Folding the $S U(2)^{N+1}$ moose chain once produces a variation of Higgsless deconstruction from Fig. 1 with $M=0$ and by renumbering $N \rightarrow 2 N$. The gauge symmetry breaking pattern is $S U(2)_{L}^{N+1} \otimes S U(2)_{R}^{N} \otimes U(1) \rightarrow U(1)_{\mathrm{em}}$, whose continuum limit has a bulk gauge group $\left[S U(2)_{L} \otimes S U(2)_{R}\right]_{5 \mathrm{D}}$.

$\mathbb{M}_{[0, \widetilde{0})}^{2}$ and the neutral gauge boson mass matrix $\mathbb{M}_{Z}^{2}=\mathbb{M}_{[0, \widetilde{0}]}^{2}$. Inspecting their eigenvalue equations we derive the consistency relations for the eigenvectors $\mathbb{X}_{n} \in$ $S U(2)_{L}$ and $\widetilde{\mathbb{X}}_{n} \in S U(2)_{R}$, related to the boundary $x^{5}=L$,

$$
\begin{aligned}
F^{2}\left(g_{N} X_{N, n}-\widetilde{g}_{N} \widetilde{X}_{N, n}\right)+f_{N+1}^{2}\left(g_{N+1} X_{N+1, n}-g_{N} X_{N, n}\right) & =0, \\
-F^{2}\left(g_{N} X_{N, n}-\widetilde{g}_{N} \widetilde{X}_{N, n}\right)+\widetilde{f}_{N+1}^{2}\left(\widetilde{g}_{N+1} \widetilde{X}_{N+1, n}-\widetilde{g}_{N} \widetilde{X}_{N, n}\right) & =0 .
\end{aligned}
$$

The eigenvectors $\left(X_{j n}, \widetilde{X}_{j n}\right)$ are related to the latticized $5 \mathrm{D}$ KK-wavefunctions $\left(\mathcal{X}_{n}\left(x_{j}^{5}\right), \widetilde{\mathcal{X}}_{n}\left(x_{j}^{5}\right)\right) \equiv\left(\mathcal{X}_{j n}, \widetilde{\mathcal{X}}_{j n}\right)=\sqrt{L}\left(\frac{g_{j}}{\widehat{g}_{L}} X_{j n}, \frac{\widetilde{g}_{j}}{\widehat{g}_{R}} \widetilde{X}_{j n}\right)$. So we rewrite (67) as conditions for $\left(\mathcal{X}_{j n}, \widetilde{\mathcal{X}}_{j n}\right)$,

$$
\begin{aligned}
F^{2}\left(\widehat{g}_{L} \mathcal{X}_{N, n}-\widehat{g}_{R} \widetilde{\mathcal{X}}_{N, n}\right)+\widehat{g}_{L} f_{N+1}^{2}\left(\mathcal{X}_{N+1, n}-\mathcal{X}_{N, n}\right) & =0, \\
-F^{2}\left(\widehat{g}_{L} \mathcal{X}_{N, n}-\widehat{g}_{R} \widetilde{\mathcal{X}}_{N, n}\right)+\widehat{g}_{R} \widetilde{f}_{N+1}^{2}\left(\widetilde{\mathcal{X}}_{N+1, n}-\widetilde{\mathcal{X}}_{N, n}\right) & =0,
\end{aligned}
$$

which are equivalent to

$$
\begin{aligned}
& \widehat{g}_{L} \mathcal{X}_{N, n}-\widehat{g}_{R} \widetilde{\mathcal{X}}_{N, n}=\widehat{g}_{L}\left(f_{N+1} / F\right)^{2}\left(\mathcal{X}_{N, n}-\mathcal{X}_{N+1, n}\right), \\
& \widehat{g}_{R}\left(\mathcal{X}_{N+1, n}-\mathcal{X}_{N, n}\right)+\zeta^{2} \widehat{g}_{L}\left(\widetilde{\mathcal{X}}_{N+1, n}-\widetilde{\mathcal{X}}_{N, n}\right)=0,
\end{aligned}
$$

where $\zeta \equiv\left(\widehat{g}_{R} \widetilde{f}_{N+1}\right) /\left(\widehat{g}_{L} f_{N+1}\right)$. With the definitions $f_{j}=h_{j} f_{L}$ and $\widetilde{f}_{j}=h_{j} f_{R}$, we have the lattice spacing $\mathbf{a} \equiv\left(2 / \widehat{g}_{L} f_{L}\right)^{2}=\left(2 / \widehat{g}_{R} f_{R}\right)^{2}$ [as shown above (23)], which leads to a geometric equality $\widehat{g}_{L} f_{L}=\widehat{g}_{R} f_{R}$. Hence the $5 \mathrm{D}$ geometry ensures $\zeta=1$. Taking continuum limit, we derive the induced 5D BCs from (69),

$$
\left.\left(\widehat{g}_{L} \widehat{A}_{L}^{a \mu}-\widehat{g}_{R} \widehat{A}_{R}^{a \mu}\right)\right|_{x^{5}=L}=0,\left.\quad \partial_{5}\left(\widehat{g}_{R} \widehat{A}_{L}^{a \mu}+\widehat{g}_{L} \widehat{A}_{R}^{a \mu}\right)\right|_{x^{5}=L}=0 .
$$

We may also reconstruct a brane term at $x^{5}=L$. This can be done by taking $f_{N}^{2} \gg f_{j<N}^{2}$ and $\widetilde{f}_{N}^{2} \gg \widetilde{f}_{j<N}^{2}$ together with $F^{2} \rightarrow \infty$. This will localize the 
diagonal group $S U(2)_{N}^{D}$ at $x^{5}=L$. By definition, $g_{j}=\widehat{g}_{L} \kappa_{j} / \sqrt{\mathbf{a}}$ and $\widetilde{g}_{j}=$ $\widehat{g}_{R} \kappa_{j} / \sqrt{\mathbf{a}}$, which contain the same $\kappa_{j}$ because of the $5 \mathrm{D}$ geometry in the continuum limit of Moose-D. Thus, $\widehat{g}_{L} / g_{N}=\widehat{g}_{R} / \widetilde{g}_{N}=\sqrt{\mathbf{a}} / \kappa_{N} \equiv \eta$. So we can write the brane coupling of $S U(2)_{N}^{D}$ as $g_{D}=1 / \sqrt{1 / g_{N}^{2}+1 / \widetilde{g}_{N}^{2}}=\eta^{-1} \widehat{g}_{L} \widehat{g}_{R} / \sqrt{\widehat{g}_{L}^{2}+\widehat{g}_{R}^{2}}$. The brane gauge field is $A_{N, D}^{a \mu}=\left(\widetilde{g}_{N} A_{N}^{a \mu}+g_{N} \widetilde{A}_{N}^{a \mu}\right) / \sqrt{g_{N}^{2}+\widetilde{g}_{N}^{2}}$. In the continuum limit, it becomes $A_{N, D}^{a \mu}=\eta\left(\widehat{g}_{R} \widehat{A}_{L}^{a \mu}+\widehat{g}_{L} \widehat{A}_{R}^{a \mu}\right) / \sqrt{\widehat{g}_{L}^{2}+\widehat{g}_{R}^{2}}$ at $x^{5}=L$, and leads to the Yang-Mills term $-\frac{1}{4}\left(F_{N, D}^{a \mu \nu}\right)^{2}=-\frac{\eta^{2}}{4\left(\widehat{g}_{L}^{2}+\widehat{g}_{R}^{2}\right)}\left[\widehat{g}_{R} \widehat{F}_{L}^{a \mu \nu}+\widehat{g}_{L} \widehat{F}_{R}^{a \mu \nu}\right]^{2}$. Hence, we derive the brane localized kinetic term at $x^{5}=L$,

$$
S_{\mathrm{BT}}=\int d^{5} \widehat{x} \delta\left(x^{5}-L\right) \frac{-\eta^{2}}{4\left(\widehat{g}_{L}^{2}+\widehat{g}_{R}^{2}\right)}\left[\widehat{g}_{R} \widehat{F}_{L}^{a \mu \nu}+\widehat{g}_{L} \widehat{F}_{R}^{a \mu \nu}\right]^{2} .
$$

The possible brane terms at $x^{5}=0$ can be similarly reconstructed as in our Moose$\mathrm{B}$ and Moose-C.

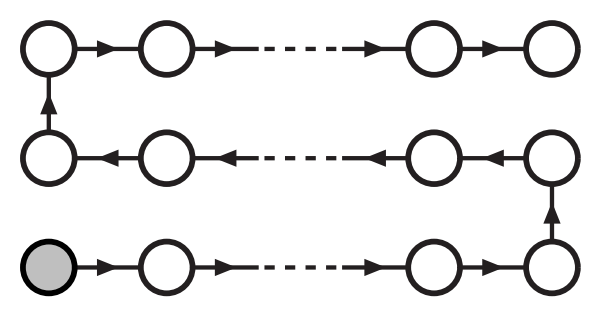

(a)

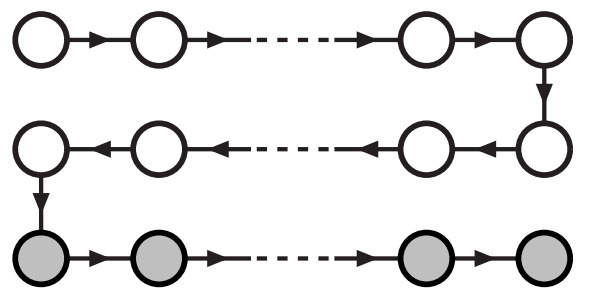

(b)

Fig. 7. Higgsless Moose-E with double-folding from the general moose theory in Fig. 1. (a). MooseE1 in the continuum limit has gauge group $\left[S U(2)^{3}\right]_{5 \mathrm{D}} \otimes U(1)_{0}$. (b). Moose-E2 in the continuum limit has gauge group $\left[S U(2)_{L} \otimes S U(2)_{R} \otimes U(1)_{X}\right]_{5 \mathrm{D}}$. They are both broken to $U(1)_{\mathrm{em}}$.

A further extension of the Moose-D is to fold the $S U(2)$-chain twice, called Moose-E1 in Fig. 7(a). This leads to a 5D continuum Higgsless theory with symmetry $\left[S U(2)^{3}\right]_{5 \mathrm{D}} \otimes U(1)_{0}$ whose BCs can be similarly derived as in Moose-D. The gauge group breaks as $\left[S U(2)^{3}\right]_{5 \mathrm{D}} \otimes U(1)_{0} \rightarrow U(1)_{\mathrm{em}}$ under compactification. Another variation is to add many $U(1)$ 's to Moose-D and form a separate $U(1)$ chain, or, from Fig. 1 we can fold the $S U(2)$-chain once in its middle as well as folding the moose at the intersection of the $S U(2)$ and $U(1)$ chains, which we call Moose-E2 in Fig.7(b). Its 5D continuum model has the bulk gauge group $\left[S U(2)_{L} \otimes S U(2)_{R} \otimes U(1)_{X}\right]_{5 \mathrm{D}}$ which, for the case of warped geometry, gives precisely the 5D Higgsless model proposed by Csáki et al [11. Possible brane terms can be generated in the continuum limit as we discussed before. With the above method, we have also derived all the induced BCs in the continuum limit of MooseE2, and found that the BCs for $\left(\widehat{A}_{L}^{a \mu}, \widehat{A}_{R}^{a \mu}, \widehat{B}^{\mu}\right)$ agree with the BCs of Csáki et al (based on the technique 21 of adding extra boundary Higgs fields with large VEV). Systematic elaboration of these will be given elsewhere.26 


\section{Effective Unitarity of Higgsless Deconstruction Without Geometry}

In this section we will demonstrate a conceptual point that the delayed unitarity violation (effective unitarity) in the general moose theory can be realized without resorting to any known $5 \mathrm{D}$ geometry. We observe that the delay of unitarity violation is essentially a collective effect due to the participation in the EWSB from many gauge groups whose own symmetry breaking scales $\left\{f_{j}\right\}$ are higher than the SM EWSB scale, $v=\left(\sqrt{2} G_{F}\right)^{-1 / 2} \simeq 246 \mathrm{GeV}$ and whose gauge couplings $\left\{g_{j}\right\}$ are larger than $g \in S U(2)_{\mathrm{SM}}$. Such a collective effect does not necessarily require any exact 5D geometry, and can be realized in general non-geometric moose settings with relevant input parameters $f_{j}>v$ and/or $g_{j}>g$.

\subsection{Formulation of the Effective Unitarity}

To analyze the unitarity it is enough to consider the $g_{N+1} \rightarrow 0$ limit, so the nontrivial part of Fig. 1 reduces to our Moose-B (Fig. 3), corresponding to the custodial symmetry limit. The other settings such as Moose-C,D,E also reduce to Moose-B at the zeroth order of $g_{N+1}$. From Eq. (11) we first derive the relevant unitary-gauge Lagrangian,

$$
\begin{aligned}
\mathcal{L} & =\sum_{j=0}^{N}-\frac{1}{4} F_{j \mu \nu}^{a} F_{j}^{a \mu \nu}+\frac{1}{2} \mathbb{A}_{\mu}^{a T} \mathbb{M}_{W}^{2} \mathbb{A}^{a \mu} \\
& =\sum_{j=0}^{N}-\frac{1}{4} \bar{W}_{j \mu \nu}^{a} \bar{W}_{j}^{a \mu \nu}+\frac{1}{2} \mathbb{W}_{\mu}^{a T} \mathbb{M}_{W}^{2}{ }^{\operatorname{diag}} \mathbb{W}^{a \mu}+\mathcal{L}_{G}^{\mathrm{int}}
\end{aligned}
$$

with the Yang-Mills interactions,

$$
\begin{aligned}
\mathcal{L}_{G}^{\mathrm{int}} & =\sum_{j=0}^{N}\left[-\frac{g_{j}}{2} C^{a b c} \bar{F}_{j}^{a \mu \nu} A_{j \mu}^{b} A_{j \nu}^{c}-\frac{g_{j}^{2}}{4} C^{a b c} C^{a d e} A_{j}^{b \mu} A_{j}^{c \nu} A_{j \mu}^{d} A_{j \nu}^{e}\right] \\
& =-\frac{G_{3}^{k m n}}{2} C^{a b c} \bar{W}_{k}^{a \mu \nu} W_{m \mu}^{b} W_{n \nu}^{c}-\frac{G_{4}^{k \ell m n}}{4} C^{a b c} C^{a d e} W_{k}^{b \mu} W_{\ell}^{c \nu} W_{m \mu}^{d} W_{n \nu}^{e}
\end{aligned}
$$

where $\bar{F}_{j}^{a \mu \nu} \equiv \partial^{\mu} A_{j}^{a \nu}-\partial^{\nu} A_{j}^{a \mu}, \bar{W}_{j}^{a \mu \nu} \equiv \partial^{\mu} W_{j}^{a \nu}-\partial^{\nu} W_{j}^{a \mu}$, and $\mathbb{W}^{a \mu}=$ $\left(W_{0}^{a \mu}, \cdots, W_{N}^{a \mu}\right)^{T}$ denotes the mass-eigenbasis fields. Also the sums over repeated indices $k, \ell, m, n=(0,1, \cdots, N)$ are implied. In Eq. (73) the effective cubic and quartic gauge couplings are given by

$$
G_{3}^{k m n}=\sum_{j=0}^{N} g_{j} \mathbb{R}_{j k}^{a} \mathbb{R}_{j m}^{b} \mathbb{R}_{j n}^{c}, \quad G_{4}^{k \ell m n}=\sum_{j=0}^{N} g_{j}^{2} \mathbb{R}_{j k}^{b} \mathbb{R}_{j \ell}^{d} \mathbb{R}_{j m}^{c} \mathbb{R}_{j n}^{e},
$$

with $\mathbb{R}^{a}=\mathbb{R}_{W}$ determined from the diagonalization $\mathbb{R}_{W}^{T} \mathbb{M}_{W}^{2} \mathbb{R}_{W}=\left(\mathbb{M}_{W}^{2}\right)^{\text {diag }}$. 
The longitudinal gauge boson scattering $W_{m L}^{a} W_{n L}^{b} \rightarrow W_{k L}^{c} W_{\ell L}^{d}$ has the following structure,

$$
\begin{aligned}
& \mathcal{T}_{m n, k \ell}^{a b, c d}=\mathcal{T}_{m n, k \ell[c]}^{a b, c d}+\mathcal{T}_{m n, k \ell[s]}^{a b, c d}+\mathcal{T}_{m n, k \ell[t]}^{a b, c d}+\mathcal{T}_{m n, k \ell[u]}^{a b, c d} \\
& =G_{4}^{m n k \ell} \mathcal{A}_{[c]}^{a b c d}+\sum_{j=0}^{N}\left[\frac{G_{3}^{m n j} G_{3}^{k \ell j}}{s-M_{W j}^{2}} \mathcal{A}_{[s]}^{a b c d}+\frac{G_{3}^{m k j} G_{3}^{n \ell j}}{t-M_{W j}^{2}} \mathcal{A}_{[t]}^{a b c d}+\frac{G_{3}^{m \ell j} G_{3}^{n k j}}{u-M_{W j}^{2}} \mathcal{A}_{[u]}^{a b c d}\right],
\end{aligned}
$$

which is a sum of possible diagrams from the contact interactions (denoted as $[c]$ ) and the gauge boson exchanges via $(s, t, u)$-channels (denoted as $[s],[t],[u])$. Here the quantities $\mathcal{A}_{[c],[s],[t],[u]}^{a b c d}$ contain an overall factor of relevant $S U(2)$ structure constants, and are functions of the energy $\sqrt{s}$ and scattering angle $\theta$, whose analytical structures are identical to that of the (Higgsless) SM (with $g^{\prime} \simeq 0$ ) up to a simple overall factor $g^{2}$. Taking the asymptotic energy $s \gg M_{W j}^{2}$ and expanding the amplitude (75), we observe that all $O\left(E^{4}\right)$ terms exactly cancel as enforced by the ET (20), while the nonzero $O\left(E^{2}\right)$ terms are suppressed by large $N+1$, leading to the delayed unitarity violation 6 in deconstruction theories.

To understand the suppressed $O\left(E^{2}\right)$ terms, it is very useful to examine the pure Goldstone interactions derived from (11), with general inputs for Moose-B,

$$
\mathcal{L}_{\widetilde{\pi}}^{\text {int }}=\frac{\mathcal{C}_{k l m n}}{6 v^{2}}\left[\left(\widetilde{\pi}_{k}^{a} \partial_{\mu} \widetilde{\pi}_{l}^{a}\right)\left(\widetilde{\pi}_{m}^{b} \partial^{\mu} \widetilde{\pi}_{n}^{b}\right)-\left(\widetilde{\pi}_{k}^{a} \widetilde{\pi}_{l}^{a}\right)\left(\partial_{\mu} \widetilde{\pi}_{m}^{b} \partial^{\mu} \widetilde{\pi}_{n}^{b}\right)\right]+O\left(\widetilde{\pi}_{j}^{6}\right),
$$

where $(k, \ell, m, n)=0,1,2, \cdots, N$ and sum over repeated indices is implied. The quartic coupling of the eaten Goldstone bosons is

$$
\mathcal{C}_{k \ell m n}=\sum_{j=1}^{N+1} \frac{v^{2}}{f_{j}^{2}} \widetilde{\mathbb{R}}_{j k}^{a} \widetilde{\mathbb{R}}_{j \ell}^{a} \widetilde{\mathbb{R}}_{j m}^{b} \widetilde{\mathbb{R}}_{j n}^{b} \leqslant 1,
$$

with $\widetilde{\mathbb{R}}^{a}=\widetilde{\mathbb{R}}_{W}$ determined from the diagonalization $\widetilde{\mathbb{R}}_{W}^{T} \widetilde{\mathbb{M}}_{W}^{2} \widetilde{\mathbb{R}}_{W}=\left(\widetilde{\mathbb{M}}_{W}^{2}\right)^{\text {diag }}$. For the special case of the Higgsless $\mathrm{SM}(N+1=M+1=1)$, the coupling $\mathcal{C}_{0000}^{\mathrm{SM}} \equiv 1$, so there is no suppression for the eaten Goldstone interactions. Computing the leading amplitude of the Goldstone scattering $\widetilde{\pi}_{n}^{a} \widetilde{\pi}_{n}^{b} \rightarrow \widetilde{\pi}_{n}^{c} \widetilde{\pi}_{n}^{d}(n \geqslant 0)$, we arrive at

$$
\mathcal{T}\left[\widetilde{\pi}_{n}^{a} \widetilde{\pi}_{n}^{b} \rightarrow \widetilde{\pi}_{n}^{c} \widetilde{\pi}_{n}^{d}\right]=\frac{\mathcal{C}_{n n n n}}{v^{2}}\left[s \delta^{a b} \delta^{c d}+t \delta^{a c} \delta^{b d}+u \delta^{a d} \delta^{b c}\right]
$$

as compared to the unsuppressed result in the Higgsless SM,

$$
\mathcal{T}\left[\widetilde{\pi}_{0}^{a} \widetilde{\pi}_{0}^{b} \rightarrow \widetilde{\pi}_{0}^{c} \widetilde{\pi}_{0}^{d}\right]_{\mathrm{SM}}=\frac{1}{v^{2}}\left[s \delta^{a b} \delta^{c d}+t \delta^{a c} \delta^{b d}+u \delta^{a d} \delta^{b c}\right] .
$$

Thus, we find that our general Moose-B has a delayed unitarity violation scale for the Goldstone scattering $\widetilde{\pi}_{n}^{a} \widetilde{\pi}_{n}^{b} \rightarrow \widetilde{\pi}_{n}^{c} \widetilde{\pi}_{n}^{d}(n \geqslant 0)$, relative to the $n=N=0$ case of the Higgsless SM,

$$
\mathscr{D}_{\mathrm{U}} \equiv \frac{E^{\star}}{E_{\mathrm{SM}}^{\star}}=\frac{1}{\sqrt{\mathcal{C}_{n n n n}}}>1, \quad \text { with } \quad \mathcal{C}_{n n n n}=\sum_{j=1}^{N+1} \frac{v^{2}}{f_{j}^{2}} \widetilde{\mathbb{R}}_{j n}^{a 4}
$$


The original analysis [6] found that for a flat-geometry input $f_{0}=\cdots=f_{N} \equiv f$ and $g_{0}=\cdots=g_{N} \equiv \widetilde{g}$, the delay factor is $\mathscr{D}_{\mathrm{U}} \simeq(f / v) \sqrt{N+1}$ for large $N+1$, where $f$ scales like $\sqrt{N+1}$ when holding $g$ and $1 / R$. This means 6 that for large $N+1$, the delay factor $\mathscr{D}_{\mathrm{U}}$ scales as $N+1$.

To analyze essential features of the delay factor $\mathscr{D}_{\mathrm{U}}$, we first consider the simplest flat version of Moose-B (Fig. 3) with massive zero-modes, by setting the inputs $f_{0}=\cdots=f_{N}=f_{N+1} \equiv f$ and $g_{0}=\cdots=g_{N} \equiv \widetilde{g}$. The Goldstone decay constants $f_{j}$ are connected to the EWSB vacuum expectation value (VEV) $v$ via the low energy four-fermion interactions, 12

$$
\left[\sum_{j=p+1}^{N+1} \frac{1}{f_{j}^{2}}\right]^{-\frac{1}{2}}=v=\left(\sqrt{2} G_{F}\right)^{-\frac{1}{2}} \simeq 246 \mathrm{GeV},
$$

where the site $j=p \in[0, N]$ is the location of left-handed SM fermions. Thus, inputting $v$ makes one of $\left\{f_{j}\right\}$ 's non-independent, and for the flat (equal) $f_{j}$ 's we deduce $f=v \sqrt{N+1-p}$. The symmetry breaking pattern also imposes a relation $g_{0}^{-2}+\cdots+g_{N}^{-2}=g^{-2}$ with $g \in S U(2)_{W}$, so for the flat (equal) $g_{j}$ 's we have $g_{j} \equiv \widetilde{g}=g \sqrt{N+1}$. With these we exactly solve all the mass eigenvalues and eigenvectors. In particular we find that site-Goldstones $\Pi^{a}=\left(\pi_{1}^{a}, \cdots, \pi_{N+1}^{a}\right)^{T}$ are connected to the eaten Goldstones $\widetilde{\Pi}^{a}=\left(\widetilde{\pi}_{0}^{a}, \cdots, \widetilde{\pi}_{N}^{a}\right)^{T}$ by the orthogonal rotation $\Pi^{a}=\widetilde{\mathbb{R}}^{a} \widetilde{\Pi}^{a}$ with

$$
\widetilde{\mathbb{R}}_{j n}^{a}=\sqrt{\frac{2}{N+\frac{3}{2}}} \sin \left[(j+1) \alpha_{n}\right], \quad(j, n=0,1, \cdots, N),
$$

where $\alpha_{n} \equiv\left(n+\frac{1}{2}\right) \pi /\left(N+\frac{3}{2}\right)$. In the gauge sector we deduce the transformation from the site-fields $\left\{A_{j}^{a \mu}\right\}$ to the mass-eigenbasis fields $\left\{W_{n}^{a \mu}\right\}$ via $\mathbb{A}^{a \mu}=\mathbb{R}^{a} \mathbb{W}^{a \mu}$ with

$$
\mathbb{R}_{j n}^{a}=\sqrt{\frac{2}{N+\frac{3}{2}}} \cos \left[\left(j+\frac{1}{2}\right) \alpha_{n}\right], \quad(j, n=0,1, \cdots, N) .
$$

Substituting (82) and the relation $f=v \sqrt{N+1-p}$ into Eq. 880, we compute the delay factor for a flat Moose-B,

$$
\mathscr{D}_{\mathrm{U}}=\frac{f}{v}\left[\sum_{j=0}^{N} \widetilde{\mathbb{R}}_{j n}^{a 4}\right]^{-\frac{1}{2}}=\left[\frac{2}{3}\left(N+\frac{3}{2}\right)(N+1-p)\right]^{\frac{1}{2}},
$$

which scales as $N / \sqrt{1.5}$ for large $N+1$ and small $p=0-O(1)$. For large $p \sim N$, the factor $\mathscr{D}_{\mathrm{U}}$ will be reduced, scaling like $\sim \sqrt{N}$.

From the above, we see that a sizable delay factor $\mathscr{D}_{\mathrm{U}}>1$ is essentially due to the collective effect of the many gauge groups participating in the EWSB via (i) the composition of the eaten Goldstone state $\widetilde{\pi}_{n}^{a}=\sum_{j} \widetilde{\mathbb{R}}_{j n}^{a} \pi_{j}^{a}$ in terms of all $N+1$ site-Goldstones $\left\{\pi_{j}^{a}\right\}$; and (ii) many comparable symmetry breaking scales $f_{j}>v$ under the condition (81). We observe that the realization of this collective 
effect does not necessarily require the moose inputs $\left(g_{j}, f_{k}\right)$ to obey any exact $5 \mathrm{D}$ geometry. In fact, for the general parameters $\left(g_{j}, f_{k}\right)$, we can achieve a visible delay factor $\mathscr{D}_{\mathrm{U}}>1$ as long as $N+1$ is reasonably large (implying that many site-Goldstones participate in the collective symmetry breaking) and all relevant $\left(g_{j}, f_{k}\right)$ are significantly larger than $(g, v)$ of the SM. With these two requirements satisfied, we find that, even if the precise pattern of the inputs $\left(g_{j}, f_{k}\right)$ are very non-geometric and random-like, a nearly maximal delay factor can be reached for each given $p$, which roughly scales like

$$
\mathscr{D}_{\mathrm{U}} \sim \mathscr{D}_{\mathrm{U}}^{\max } \longrightarrow O(1)[(N+1)(N+1-p)]^{\frac{1}{2}} .
$$

Finally, we note that the above instructive estimate of the delay factor $\mathscr{D}_{\mathrm{U}}$ is based on the leading Goldstone amplitude at $O\left(E^{2}\right)$. The exact longitudinal gauge boson amplitude (75) may result in a stronger unitarity limit $E^{\star}$ and thus smaller delay factor $\mathscr{D}_{\mathrm{U}}$, since the analysis of leading $E^{2}$-amplitude of the Goldstone scattering has ignored all the subleading terms of $O\left(E^{0}\right)$ and $O\left(M_{W n}^{2} / E^{2}\right)$ which could be visible when $N+1$ is not too large and the limit $E^{\star}$ is only around a few $\mathrm{TeV}$. So, in the explicit analysis below, we will directly compute the gauge boson amplitude (75) for deriving the unitarity bound $E^{\star}$.

\subsection{Explicit Analysis of Effective Unitarity Without Geometry}

Next, we explicitly demonstrate how the effective unitarity can be realized in various minimal moose theories without geometry. For the explicit analysis, we will focus on the zero-mode amplitude $\mathcal{T}\left[W_{0 L}^{a} W_{0 L}^{b} \rightarrow W_{0 L}^{c} W_{0 L}^{d}\right]$ which is also to be tested at the LHC. The unitarity requires its $s$-wave amplitude to be bounded from above, $\left|a_{0000}^{0}[a b, c d]\right|<1 / 2$, where the possible effect of final state identical particles can be included 23 . We know that the naive Higgsless SM $(N+1=$ $M+1=1)$ predicts the unitarity violation scale in the $W_{0 L} W_{0 L}$ scattering as, $E_{\mathrm{SM}}^{\star} \simeq \sqrt{8 \pi} v \simeq 1.2 \mathrm{TeV} 102423$. This is significantly lower than the conventional cutoff scale $\Lambda \simeq 4 \pi v \simeq 3 \mathrm{TeV}$ as estimated by the consistency of chiral perturbation theory 25. So we will identify and analyze the minimal moose (MM) models that can exhibit an effective unitarity at least up to a scale $E^{*} \gtrsim 3 \mathrm{TeV}$.

We perform the numerical analysis at the zeroth order of $U(1)$ coupling $g_{N+1}$. We ensure the light mass $M_{W}=M_{W 0} \simeq 80 \mathrm{GeV}$ and the lowest new gauge boson mass $M_{W 1} \gtrsim 800 \mathrm{GeV}$ by the phenomenological consideration. We will consider one minimal moose $S U(2)^{3} \otimes U(1)$ (called MM3, $N+1=3$ and $M=0$ ) with the left(right)-handed fermions coupled to the site $j=p=0(j=q=3)$ in Fig. 1, and another minimal moose (called MM4, $N+1=4$ and $M=0$ ) with the left(right)handed fermions coupled to the site $j=p=1(j=q=4)$. We compute the unitarity limit $E^{\star}$ for each model by scanning the parameter space in Fig. 8, where the $E^{\star}$ with "non-geometric" inputs (of random-like pattern) are shown by solid curves and are compared to the "geometric" inputs (with relevant $g_{j}$ 's and/or $f_{j}$ 's being equal) as depicted by dotted curves. We have chosen the scattering $W_{0 L}^{+} W_{0 L}^{-} \rightarrow W_{0 L}^{+} W_{0 L}^{-}$ 
in Fig. 8 for illustration since other channels are found to exhibit similar features. For this process the $u$-channel term in (75) is absent. [The exact tree-level amplitude of this scattering in the Higgsless SM $(N+1=M+1=1)$ may be found in Eq. (3.17) of the first paper in [15] which does not pose delayed unitarity violation.]

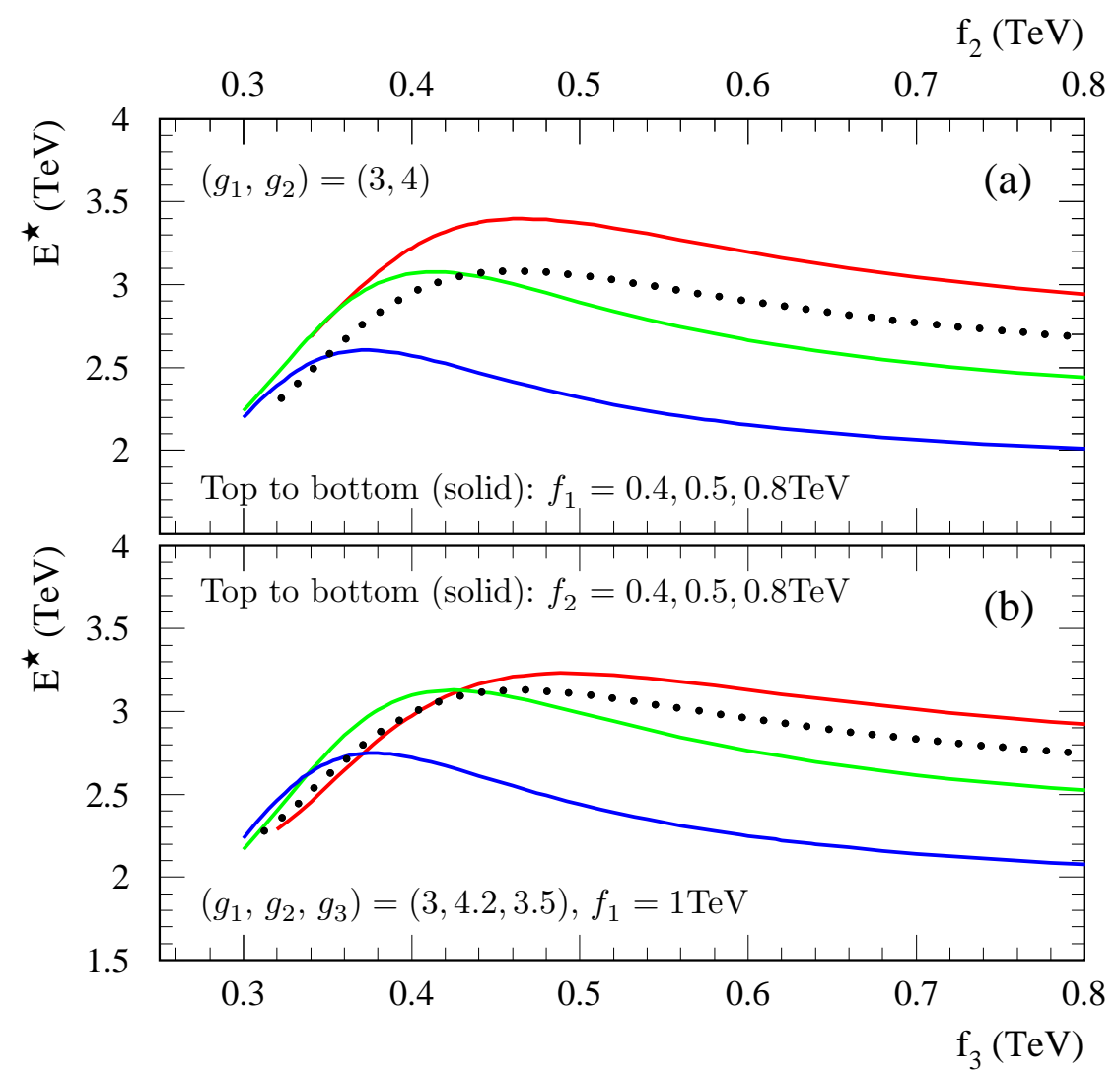

Fig. 8. Unitarity limit $E^{\star}$ is shown for the moose $S U(2)^{3} \otimes U(1)$ (MM3) with $p=0$ in plot (a) as a function of $f_{2}$, and for the moose $S U(2)^{4} \otimes U(1)$ (MM4) with $p=1$ in plot (b) as a function of $f_{3}$. The solid curves are shown for three sets of non-geometric inputs which appear random-like. As a comparison, the dotted curves come from two sets of typical geometric inputs: $g_{1}=g_{2}=4$, $f_{1}=\sqrt{3} v \simeq 0.43 \mathrm{TeV}$ in plot (a); and $g_{1}=g_{2}=g_{3}=4, f_{1}=1 \mathrm{TeV}, f_{2}=\sqrt{3} v$ in plot (b).

Fig. 8 shows that either solid or dotted curves are generally quite flat and there exists no sharp "peak" when we vary $f_{2}$ in the plot (a) and $f_{3}$ in the plot (b). There is a sizable region on each curve to realize a nearly maximal scale $E^{\star} \sim E_{\max }^{\star}$. The inputs for dotted curves mimic closely for certain flat-geometry settings, but for realizing $E^{\star} \sim E_{\max }^{\star}$ they clearly do not exhibit any real advantage over other nongeometric inputs (shown as solid curves). We also find $E^{\star}$ to be less sensitive to the variation of the pattern of gauge couplings. To have a direct feeling, we explicitly list two sample inputs/outputs for each model. For the MM3 model with $p=0$, in- 
putting $\left(g_{1}, g_{2}\right)=(3.1,4)$ and $\left(f_{1}, f_{2}\right)=(0.5,0.4) \mathrm{TeV}$, we derive the gauge boson mass-spectrum $\left(M_{W 0}, M_{W 1}, M_{W 2}\right) \simeq(0.08,0.8,1.3) \mathrm{TeV}$, and the unitarity violation scale $E^{\star} \simeq 3.1 \mathrm{TeV}$. For the MM4 model with $p=1$, inputting $\left(g_{1}, g_{2}, g_{3}\right)=$ $(3,4.2,3.5)$ and $\left(f_{1}, f_{2}, f_{3}\right)=(1,0.5,0.38) \mathrm{TeV}$, we derive the gauge boson massspectrum $\left(M_{W 0}, M_{W 1}, M_{W 2}, M_{W 3}\right) \simeq(0.08,0.81,1.3,1.8) \mathrm{TeV}$, and the unitarity violation scale $E^{\star} \simeq 3.0 \mathrm{TeV}$; if we reduce the input values of $\left(g_{1}, g_{2}\right)$ to $\left(g_{1}, g_{2}\right)=(2.5,4)$ and keep other inputs unchanged, we obtain a very similar mass-spectrum $\left(M_{W 0}, M_{W 1}, M_{W 2}, M_{W 3}\right) \simeq(0.08,0.8,1.2,1.6) \mathrm{TeV}$ and a unitarity limit $E^{\star} \simeq 3.1 \mathrm{TeV}$.

We have also analyzed other moose models with different inputs of $N+1$ and/or $p$ and found similar features, although the limit $E^{\star}$ for each scattering process generally increases for larger $N+1$ and smaller $p$, as expected. More systematic analysis including the effect of delocalized fermions will be given elsewhere.26

\section{Discussion and Conclusion}

In conclusion, using the powerful deconstruction approach has revealed that $4 \mathrm{D}$ gauge theories exhibit far richer dynamics than naive expectation. With this conceptually clean tool, we have formulated and classified the compactified 5D gauge symmetry breaking (without/with gauge group rank reduction) via spontaneous symmetry breaking in general gauge-invariant $4 \mathrm{D}$ moose theories. We demonstrate that various consistent 5D boundary conditions (BCs) as well as possible brane terms are automatically induced from taking proper continuum limits of the general gauge-invariant moose theory.

We observe that the effective unitarity (delayed unitarity violation) is essentially a collective effect due to the participation in the EWSB from many gauge groups whose symmetry breaking scales $\left(f_{j}\right)$ are higher than the SM EWSB scale $v=\left(\sqrt{2} G_{F}\right)^{-1 / 2}$ and whose gauge couplings $g_{j}$ are larger than the SM $S U(2)_{W}$ coupling $g$. We find that the effective unitarity can be naturally realized in a wide class of $4 \mathrm{D}$ moose theories even with a few extra gauge bosons, without resorting to any known 5D geometry. As shown in Sec. 5, an important advantage of the advocated non-geometric moose theories is that they contain much larger parameter space than the special geometric setting, so they open up much wider windows for accommodating experimental constraints while retaining effective unitarity. More elaboration including the effect of delocalized fermions will be given elsewhere 26 .

The precision constraints on our general moose theory (Fig. 1) with the SM fermions localized at arbitrary sites $j=p(0 \leqslant p \leqslant N)$ and $j=q(N+1 \leqslant q \leqslant$

$K+1)$ have been systematically analyzed 12 . In particular, by requiring a sizable gap between the light masses $M_{W, Z}$ and the first heavy masses $M_{W^{\prime}, Z^{\prime}}(\lesssim \sqrt{8 \pi} v)$, a sum rule for the precision parameters (corresponding to $\widehat{S}$ ) can be derived, 12

$$
\widehat{S}=\frac{1}{4 s_{Z}^{2}}\left[\alpha S+4 c_{Z}^{2}(\Delta \rho-\alpha T)+\frac{\alpha \delta}{c_{Z}^{2}}\right] \geqslant \sum_{r=p+1}^{N} \frac{M_{W}^{2}}{M_{r}^{2}} \gtrsim \frac{M_{W}^{2}}{8 \pi v^{2}} \simeq 4 \times 10^{-3},
$$


where $p \in[0, N)$ and $\left\{M_{r}^{2}\right\}$ are eigenvalues of mass matrix $\mathbb{M}_{(p, N+1)}^{2}$. Although the precision data seem not to favor (86), this phenomenological constraint may be evaded in a number of ways, e.g., by smearing the fermion location out of a single site in the general moose theory, 26 similar to the use of delocalized bulk fermions in certain $5 \mathrm{D}$ models 27 .

Acknowledgments I am grateful to R. S. Chivukula, E. H. Simmons, M. Tanabashi and M. Kurachi for fruitful collaborations and helpful suggestions to the manuscript. I also thank D. A. Dicus, A. Hebecker, R. Sundrum, J. Terning and U. Varadarajan for useful discussions. This work was supported by U.S. Department of Energy under grant No. DE-FG03-93ER40757.

\section{References}

1. N. Arkani-Hamed, A. G. Cohen, H. Georgi, Phys. Rev. Lett. 86, 4757 (2001);

C. T. Hill, S. Pokorski, J. Wang, Phys. Rev. D64, 105005 (2001).

[For some related studies in the pre-deconstruction era, e.g., M. B. Halpern and W. Siegel, Phys. Rev. D11, 2967 (1975); T. Eguchi and H. Kawai, Phys. Rev. Lett. 48, 1063 (1982). I thank M. B. Halpern for drawing my attention to his early paper.]

2. H. Georgi, Nucl. Phys. B266, 274 (1986).

3. W. A. Bardeen, R. B. Pearson and E. Rabinovici, Phys. Rev. D21, 1037 (1980).

4. C. G. Callen, S. Coleman, J. Wess, and B. Zumino, Phys. Rev. 177, 2247 (1969).

5. T. Kaluza, Sitz. Preuss Akad. Wiss K 1 (1921) 966; O. Klein, Z. Phys. 37 (1926) 895.

6. R. S. Chivukula and H.-J. He, Phys. Lett. B532 (2002) 121 hep-ph/0201164.

7. R. S. Chivukula, D. A. Dicus, H.-J. He, S. Nandi, in proceedings of the international conference of SUSY-2003, Tucson, Arizona, USA, June 5-10, 2003 hep-ph/0402222.

8. R. S. Chivukula, D. A. Dicus, H.-J. He, Phys. Lett. B 525, 175 (2002) hep-ph/0111016; R. S. Chivukula, D. A. Dicus, H.-J. He, and S. Nandi, Phys. Lett. B562, 109 (2003) hep-ph/0302263.

9. P. W. Higgs, Phys. Lett. 12, 132 (1964).

10. D. A. Dicus and V. S. Mathur, Phys. Rev. D7, 3111 (1973); B. W. Lee, C. Quigg and H. B. Thacker, Phys. Rev. D16, 1519 (1977); M. J. G. Veltman, Acta Phys. Polon. B8, 475 (1977).

11. C. Csaki, C. Grojean, H. Murayama, L. Pilo and J. Terning, Phys. Rev. D69, 055006 (2004); C. Csaki, C. Grojean, L. Pilo and J. Terning, Phys. Rev. Lett. 92, 101802 (2004); G. Cacciapaglia, C. Csaki, C. Grojean, J. Terning, Phys. Rev. D70, 075014 (2004), and hep-ph/0409126 Y. Normura, JHEP 11, 050 (2003); R. Barbieri, A. Pomarol and R. Rattazzi, Phys. Lett. B591, 141 (2004); hep-ph/0405040 T. Ohl and C. Schwinn, Phys. Rev. D70, 045019 (2004); R. Foadi, S. Gopalkrishna, C. Schmidt, JHEP 03, 042 (2004); hep-ph/0409266 H. Davoudiasl, J. L. Hewett, B. Lillie, T. G. Rizzo, Phys. Rev. D70, 015006 (2004); J. L. Hewett, B. Lillie, T. G. Rizzo, JHEP 0410, 014 (2004); R. S. Chivukula, E. H. Simmons, H.-J. He, M. Kurachi, M. Tanabashi, Phys. Rev. D70, 075008 (2004); Phys. Lett. B603, 210 (2004); S. Gabriel, S. Nandi, G. Seidl, hep-ph/0406020 N. Evans and P. Membry, hep-ph/0406285 M. Papucci, hep-ph/0408059 H. Georgi, hep-ph/0408067 M. Perelstein, hep-ph/0408072 and references therein.

12. R. S. Chivukula, E. H. Simmons, H.-J. He, M. Kurachi, and M. Tanabashi, Phys. Rev. D (in press) hep-ph/0410154.

13. K. Sfetsos, Nucl. Phys. B612, 191 (2001) hep-th/0106126. 
14. C. Becchi, A. Rouet and B. Stora, Ann. Phys. 98, 287 (1976); Commun. Math. Phys. 42, 127 (1975); I. V. Tyutin, Lebedev institute preprint N39 (1975).

15. H.-J. He and W. B. Kilgore, Phys. Rev. D55, 1515 (1997); H.-J. He, Y.-P. Kuang, C.P. Yuan, Phys. Rev. D51, 6463 (1995); H.-J. He, Y.-P. Kuang, X. Li, Phys. Lett. B329, 278 (1994); Phys. Rev. D49, 4842 (1994); Phys. Rev. Lett. 69, 2619 (1992); for a comprehensive review, H.-J. He, Y.-P. Kuang, C.-P. Yuan, DESY-97-056 hep-ph/9704276, and references therein.

16. E.g., H. Abe, T. Kobayashi, N. Maru, K. Yoshioka, Phys. Rev. D67, 045019 (2003); A. Falkowski and H. D. Kim, JHEP 0208, 052 (2002); L. Randall, Y. Shadmi, N. Weiner, JHEP 0301, 055 (2003).

17. L. Randall and R. Sundrum, Phys. Rev. Lett. 83, 3370 (1999).

18. L. Randall and M. D. Schwartz, JHEP 0111, 003 (2002) hep-th/0108114.

19. H.-J. He, et al., Phys. Rev. D55, 3038 (1997); Phys. Lett. B382, 149 (1996).

20. B. W. Lee, Gauge Theories, in Methods in Field Theory, eds. R. Balian and J. ZinnJustin (North-Holland, Amsterdam, 1976).

21. A. Hebecker and J. March-Russell, Nucl. Phys. B625, 128 (2002) hep-ph/0107039.

22. R. S. Chivukula, H.-J. He, J. Howard, and E. H. Simmons, Phys. Rev. D 69, 015009 (2004) hep-ph/0307209.

23. E.g., D. A. Dicus and H.-J. He, hep-ph/0409131

24. M. S. Chanowitz and M. K. Gaillard, Nucl. Phys. B261, 379 (1985).

25. A. Manohar and H. Georgi, Nucl. Phys. B234, 189 (1984).

26. R. S. Chivukula, et al., in preparation.

27. G. Cacciapaglia, et al., in [1]; R. Foadi, et al., in [11.

28. A. Muck, L. Nilse, A. Pilaftsis, R. Ruckl, hep-ph/0411258 